ARTICLE

\title{
Genome-wide functional analysis of phosphatases in the pathogenic fungus Cryptococcus neoformans
}

Jae-Hyung Jin (10 1,7, Kyung-Tae Lee (1) 1,7, Joohyeon Hong${ }^{1}$, Dongpil Lee ${ }^{1}$, Eun-Ha Jang ${ }^{1}$, Jin-Young Kim (1) ${ }^{1}$, Yeonseon Lee ${ }^{1}$, Seung-Heon Lee (1) 1, Yee-Seul So ${ }^{1}$, Kwang-Woo Jung (1) 1,6, Dong-Gi Lee (1) 1, Eunji Jeong ${ }^{1}$,

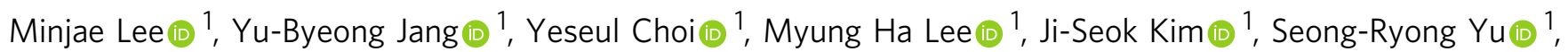
Jin-Tae Choi (1) ', Jae-Won La', Haneul Choi', Sun-Woo Kim¹, Kyung Jin Seo', Yelin Lee (1) 1, Eun Jung Thak², Jaeyoung Choi (i) ${ }^{3}$, Anna F. Averette ${ }^{4}$, Yong-Hwan Lee (i) ${ }^{5}$, Joseph Heitman ${ }^{4}$, Hyun Ah Kang ${ }^{2}$, Eunji Cheong (i] ${ }^{1} \&$ Yong-Sun Bahn (i]) ${ }^{1 凶}$

Phosphatases, together with kinases and transcription factors, are key components in cellular signalling networks. Here, we present a systematic functional analysis of the phosphatases in Cryptococcus neoformans, a fungal pathogen that causes life-threatening fungal meningoencephalitis. We analyse 230 signature-tagged mutant strains for 114 putative phosphatases under 30 distinct in vitro growth conditions, revealing at least one function for 60 of these proteins. Large-scale virulence and infectivity assays using insect and mouse models indicate roles in pathogenicity for 31 phosphatases involved in various processes such as thermotolerance, melanin and capsule production, stress responses, O-mannosylation, or retromer function. Notably, phosphatases Xpp1, Ssu72, Siw14, and Sit4 promote blood-brain barrier adhesion and crossing by $\mathrm{C}$. neoformans. Together with our previous systematic studies of transcription factors and kinases, our results provide comprehensive insight into the pathobiological signalling circuitry of $C$. neoformans.

\footnotetext{
${ }^{1}$ Department of Biotechnology, College of Life Science and Biotechnology, Yonsei University, Seoul 03722, Korea. ${ }^{2}$ Department of Life Science, College of Natural Science, Chung-Ang University, Seoul 06974, Korea. ${ }^{3}$ Smart Farm Research Center, Korea Institute of Science and Technology, Gangneung 25451 , Korea. ${ }^{4}$ Departments of Molecular Genetics and Microbiology, Medicine, and Pharmacology and Cancer Biology, Duke University Medical Center, Durham, NC 27710, USA. ${ }^{5}$ Department of Agricultural Biotechnology, Seoul National University, Seoul 08826, Korea. ${ }^{6}$ Present address: Radiation Research Division, Korea Atomic Energy Research Institute, Jeongeup 56212, Korea. ${ }^{7}$ These authors contributed equally: Jae-Hyung Jin, Kyung-Tae Lee.

凶email: ysbahn@yonsei.ac.kr
} 
A Il living organisms employ complex signalling pathways for dynamic response to changes in their environment to survive and proliferate. When an environmental cue occurs, an organism receives the signal through a sensor (i.e., receptor protein), which subsequently activates downstream effectors to counteract the incoming stress. Upon resolution of or adaptation to the cue, the signalling pathway is generally desensitized in a timely manner in order to be reactivated later. This process of desensitization is governed by phosphorylation and dephosphorylation, events modulated by kinases and phosphatases, respectively, which constitute key post-translational modifications that switch signalling components on or off. Thus, orchestrated regulation of kinases and phosphatases in signalling pathways are critical to maintaining cellular homoeostasis ${ }^{1}$.

Fungal pathogens also utilize these complex signalling pathways to respond and adapt to dramatic environmental changes occurring during infection, colonization, proliferation, and dissemination to various tissues within a host. Although several key signalling pathways governing fungal pathogenicity have been elucidated in past decades ${ }^{2}$, recent large-scale functional analyses of fungal kinases and transcription factors (TFs) provide even more comprehensive insights into how fungal signalling pathways modulate infection and virulence. Indeed, $178 \mathrm{TFs}$ and 183 kinases were identified in Cryptococcus neoformans, which causes life-threatening meningoencephalitis mainly in immunocompromised patients and is responsible for more than 180,000 deaths annually ${ }^{3}$; of the 155 and 129 , respectively, functionally characterized in vitro and in vivo ${ }^{4,5}, 45 \mathrm{TFs}$ and 63 kinases are involved in its pathogenicity. Nevertheless, the regulation of these signalling components and their coordination in pathogenicity remain elusive.

To better understand TF and kinase networks, co-analysis of the phosphatase networks as signalling counterparts is essential. Historically, phosphatases have received less attention than kinases, probably because kinases, which possess high substrate specificity, are generally considered to be better drug targets than substrate-promiscuous phosphatases ${ }^{6}$. However, recent systematic functional phosphatome data in several fungal pathogens indicate that phosphatases play critical roles in maintaining cellular homoeostasis by controlling growth and cell cycle, differentiation, stress response, and metabolism. In ascomycete fungi, genome-wide analyses of phosphatases have identified 32 protein phosphatases and functionally characterized 24 of them in Aspergillus fumigatus, the majority of which contribute to stress response, iron assimilation, and toxin production and resistance ${ }^{7}$. Furthermore, in the wheat scab fungus Fusarium graminearum, Yun et al. identified 82 phosphatase genes, disrupted 71 of them, and functionally analysed these mutants across 15 phenotypic traits (e.g., growth, nutrient response, and virulence), finding that 25 of these phosphatases are involved in virulence of the phytopathogenic fungus ${ }^{8}$. Among phosphatases, calcineurin is a wellestablished drug target and a globally conserved fungal virulence factor'.

The goal of this study was to systematically analyse the functions of the $C$. neoformans phosphatase network and interplay with its kinase and TF networks to better understand its pathobiological signalling. To this end, we constructed a high-quality library of 219 signature-tagged gene-deletion mutant strains representing 109 phosphatases out of 139 putative phosphatases identified in C. neoformans, in addition to 11 signature-tagged mutants representing six phosphatases that we previously constructed $^{4,5,10,11}$. Using a total of 230 signature-tagged mutants representing 114 phosphatases, we analysed their phenotypic traits under 30 distinct in vitro conditions and performed a largescale virulence assay using two model host systems (insect and murine). This entire phosphatase phenome data set is freely available to the public through the C. neoformans Phosphatase Phenome Database (http://phosphatase.cryptococcus.org).

\section{Results}

Identification of phosphatases in C. neoformans. To select putative phosphatase genes, we surveyed a curated annotation of phosphatases in the FungiDB C. neoformans (H99 strain) genome database (http://fungidb.org/fungidb) and validated the presence of phosphatase-related domains using protein sequence analysis and classification (Interpro). Through these analyses, we retrieved a total of 139 putative phosphatase genes in C. neoformans (Fig. 1a, b and Supplementary Data 1). We made three notable findings. First, phosphatases are generally less evolutionarily conserved than kinases, but more conserved than TFs (Supplementary Fig. 1 and Supplementary Data 2). Second, despite a paucity of tyrosine kinases [only three tyrosine kinase-like (TKL) proteins], C. neoformans contains 21 protein tyrosine phosphatases (PTPs). Third, compared to other non-pathogenic and pathogenic yeasts, C. neoformans contains a similar number of putative phosphatases (Fig. 1c and Supplementary Data 3).

Construction of the $C$. neoformans phosphatase mutant library. To obtain comprehensive insights into the $C$. neoformans phosphatome networks and their biological functions, we constructed gene-deletion mutants for each phosphatase gene and analysed their in vitro and in vivo phenotypic traits. Among the 139 putative phosphatase genes, 15 have been functionally characterized previously by gene-deletion studies $\left(P T P 1^{10}, P T P 2^{10}\right.$ $Y S A 1^{12}, C N A 1^{13}, C A C 1^{11}, T P S 2^{14}, C C R 4^{15}, H A D 1^{16}, E P P 1^{17}$, $X P P 1^{17}, A P H 1^{18}, A S P 1^{19}, I S C 1^{20}, P P G 1^{21}$, and $\left.P P H 3^{21}\right)$. In addition, we previously deleted two phosphatase genes with kinase domains (OXK1 and FBP26) for construction of the kinase mutant library ${ }^{5}$ and one phosphatase gene with a DNA-binding domain (APN2) for the TF mutant library ${ }^{4}$. Besides the 11 signature-tagged mutant strains (two for each PTP1, PTP2, $O X K 1, F B P 26$, and APN2 and one for CAC1) that we previously constructed, we performed a large-scale homologous recombination-based gene deletion using nourseothricinresistance markers containing a series of unique oligonucleotide signature tags that we previously employed for construction of the TF and kinase mutant libraries ${ }^{4,5}$ (Supplementary Data 4). To obtain a high-quality phosphatase mutant library, we constructed more than two independent mutants for each gene and verified their genotypes by diagnostic PCR and Southern blot analysis. As a result, here, we report 219 new mutant strains representing 109 phosphatases and the analysis of a total of 230 mutant strains representing 114 phosphatases (Supplementary Data 5). Disruption strategies, primer sequences, Southern blot results, and mutant phenome data are available in the Cryptococcus neoformans Phosphatase Phenome Database (http://phosphatase. cryptococcus.org) we constructed for this study. For the remaining 25 phosphatase genes, we failed to obtain any viable transformants or obtained only potential aneuploid mutants possessing both wild-type and mutant alleles after repeated attempts, suggesting these genes may be essential (Supplementary Data 6). We also developed the Cryptococcus neoformans Phenome Gateway Database (http://www.cryptococcus.org/), in which every TFs, kinases, and phosphatases studied ${ }^{4,5}$ are linked to the most widely used fungal genome database "FungiDB" (https://fungidb.org/fungidb/) to maximize connectivity between research data.

Phenotypic and in vivo expression profiling of phosphatome. To elucidate the functions of $C$. neoformans phosphatases deleted in this and previous studies, we examined phenotypic traits under 
a

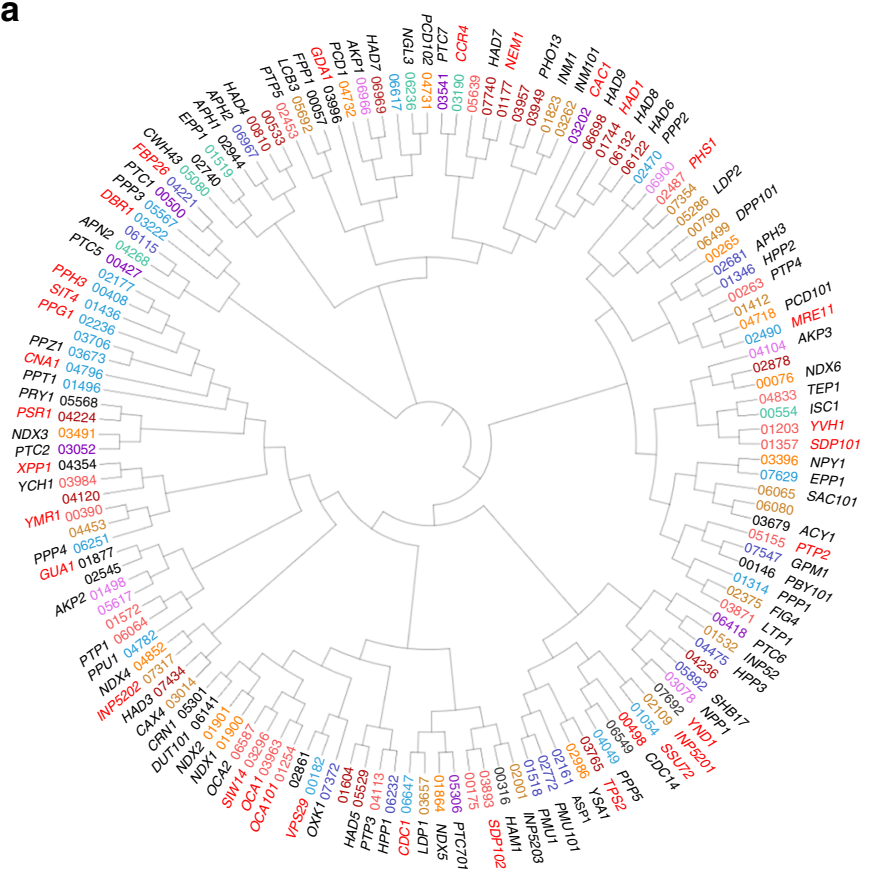

b

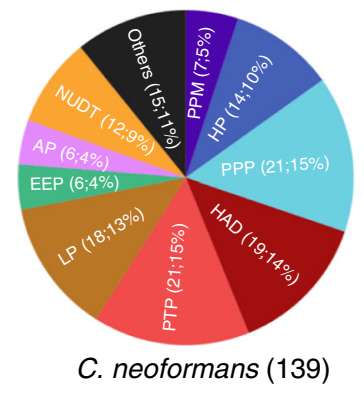

Metallo-dependent phosphatase (PPM)

Histidine phosphatase (HP)

Phosphoprotein phosphatase (PPP)

Haloacid dehalogenase (HAD)

Protein tyrosine phosphatase (PTP)

Lipid phosphatase (LP)

Endonuclease exonuclease phosphatase (EEP)

Alkaline phosphatase (AP)

NUDIX hydrolase (NUDT)

Other phosphatase

(fructose-1,6-bisphosphatase, histidinol

phosphatase, nucleoside phosphatase,

Thiamine triphophatase, pyrophosphatase,

exopolyphosphatase, acylphosphatase)
C

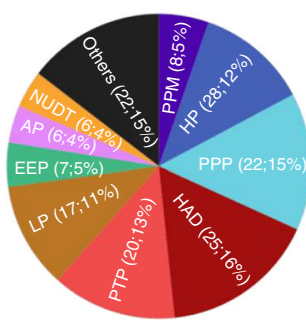

S. cerevisiae (151)

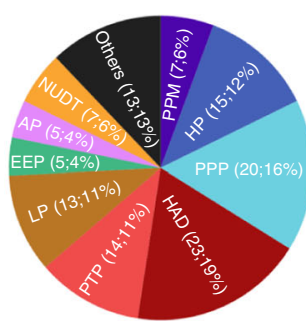

S. pombe (124)

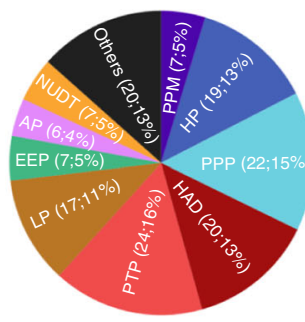

C. albicans (149)

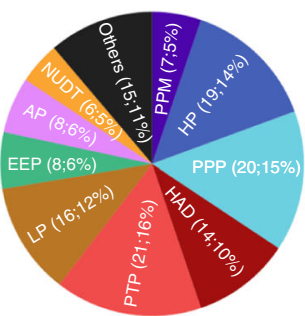

U. maydis (133)

Fig. 1 Classification of phosphatases in C. neoformans and other fungal species. a Phylogenetic analysis of phosphatases utilized a protein sequencebased alignment in ClustalX2 (Science Foundation Ireland, Dublin, Ireland). These alignment data were used to illustrate the phylogenetic tree through a web-based drawing application (Interactive Tree Of Life, https://itol.embl.de). Red letters represent pathogenicity-related phosphatases in C. neoformans identified from 114 phosphatase gene-deletion mutants that we constructed. b Pie chart indicating the classification and distribution of 139 phosphatases based on phosphatase-related domain, as analysed by using the InterPro Database. c Pie chart indicating the distribution of putative phosphatase genes in the fungal species S. cerevisiae, S. pombe, C. albicans, and U. maydis surveyed and classified using the same strategies as for C. neoformans.

30 distinct in vitro conditions: growth at different temperatures $\left(25,30,37\right.$, and $\left.39^{\circ} \mathrm{C}\right)$, mating efficiency, virulence factor production (capsule, melanin, and urease), stress responses (osmotic/ cation salt, oxidative, genotoxic, ER, cell membrane/wall, and heavy metal stresses), and antifungal drug susceptibility (Supplementary Data 7). The whole phenome data set of the phosphatase mutant collection was qualitatively illustrated with a colour scale (Fig. 2 and Supplementary Data 7). This systematic phenotypic analysis revealed that $\sim 53 \%$ of the phosphatase mutants (60/114) showed at least one discernible phenotype (Fig. 2); $72 \%$ of these (43/60) have not been previously functionally analysed. When we compared our phosphatase phenome data with their corresponding BLAST matrix data (Supplementary Fig. 2 and Supplementary Data 2), we found that the putative essential phosphatases that we could not disrupt and the phosphatases showing multiple phenotypic traits were generally more evolutionarily conserved. Phenotypic clustering of phosphatase mutants revealed groups of phosphatases that could be directly or indirectly correlated with regards to cellular function (Fig. 2).

In addition, we monitored how each of the 139 phosphatase genes were transcriptionally regulated during murine infection by assessing expression levels of each phosphatase gene in recovered tissues (lungs, brain, spleen, and kidneys) after 3, 7, 14, and 21 days post-infection (dpi) with strain H99S using the nCounter gene expression profile (NanoString, Seattle, WA, USA) with 139 novel phosphatase probes. The in vivo expression levels of each phosphatase were normalized to average in vivo expression levels of eight housekeeping genes (mitochondrial protein, CNAG_00279; microtubule binding protein, CNAG_00816; aldose reductase, CNAG_02722; cofilin, CNAG_02991; actin, CNAG_00483; tubulin $\beta$ chain, CNAG_01840; tubulin $\alpha-1 \mathrm{~A}$ chain, CNAG_03787; histone H3, CNAG_04828) and compared to those under basal growth conditions [yeast extract-peptonedextrose (YPD) medium at $30^{\circ} \mathrm{C}$ ] (Fig. 2, Supplementary Fig. 3, and Data 8). Notably, we detected increased in vivo expression of numerous phosphatase genes in the brain, kidneys, and spleen during early infection (3-7 dpi; Fig. 2). Normally, cryptococcal CFUs are barely detected in the brain and other organs, except the lungs, during early infection stage in the intranasal inhalation infection model, partly due to the detection limit of the conventional fungal burden assay. However, we used the Nanostring nCounter platform, which can detect a single gene transcript without amplification 22,23 . Therefore, once a small number of $C$. neoformans cells are hematogenously disseminated 


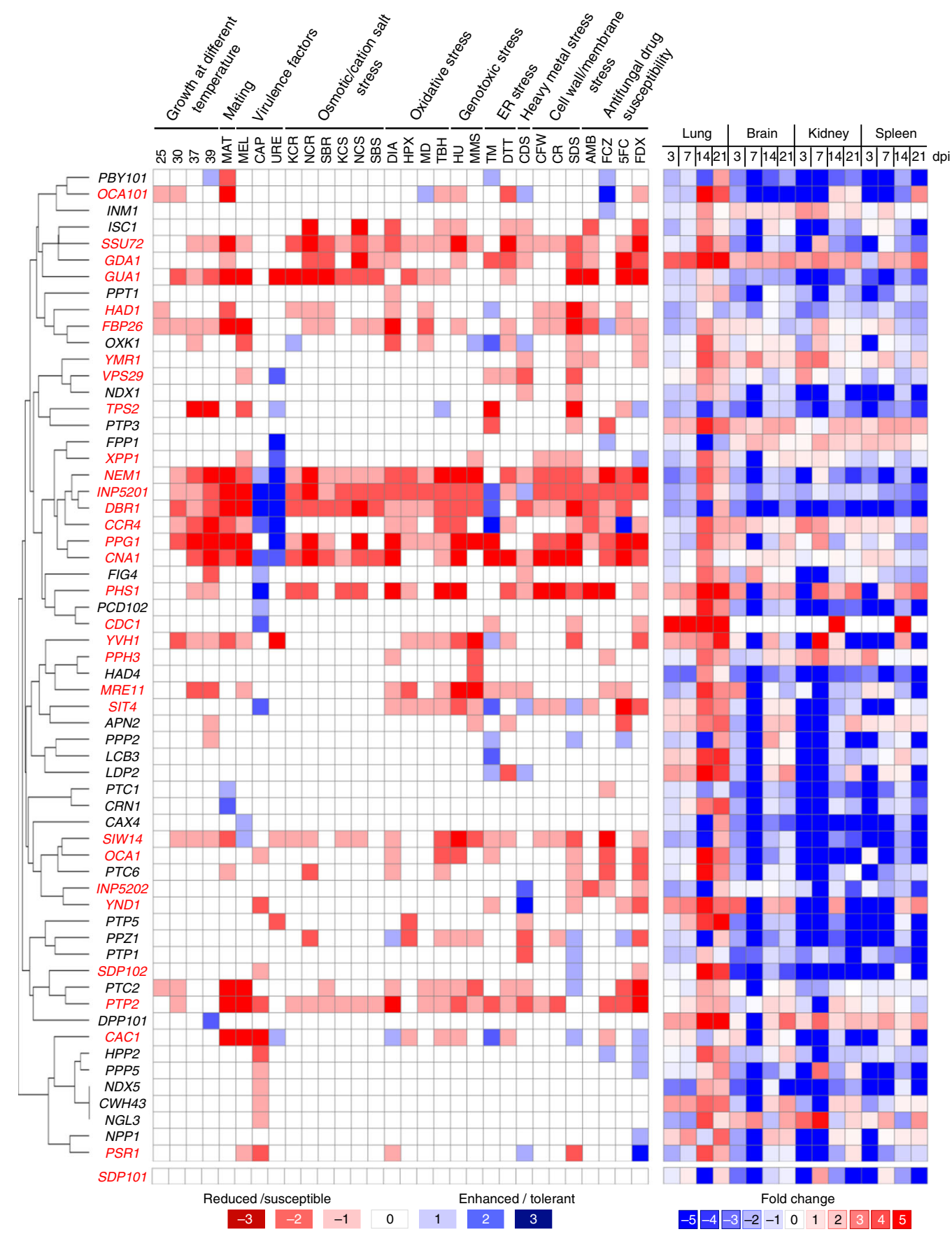

Fig. 2 Phenotypic clustering and in vivo expression profiling of phosphatases in C. neoformans. In vitro phenotypic traits were examined under 30 different growth conditions and scored on a 7-point scale ( -3 : strongly reduced/susceptible, -2 : moderately reduced/susceptible, -1 : weakly reduced/ susceptible, 0 : wild-type like, +1 weakly enhanced/tolerant, +2 : moderately enhanced/tolerant, +3 : strongly enhanced/tolerant). All phenotypic data are available in the Cryptococcus neoformans Phosphatase Phenome Database (http://phosphatase.cryptococcus.org). More than three biologically independent experiments were performed for each phenotypic trait. Hierarchical phenotypic clustering of 60 phosphatases showing at least one phenotypic trait was performed with one minus Pearson correlation in Morpheus (https://software.broadinstitute.org/morpheus). The right panel shows the corresponding in vivo gene expression profiles for each phosphatase gene determined by NanoString nCounter platform analysis during intranasal murine infection with $C$. neoformans. Red letters represent pathogenicity-related phosphatases. The sdp1010 mutant, which did not show any in vitro phenotypes but exhibited reduced infectivity, was also included. Abbreviations: $2525^{\circ} \mathrm{C}, 3030^{\circ} \mathrm{C}, 3737^{\circ} \mathrm{C}, 3939^{\circ} \mathrm{C}, \mathrm{CAP}$ capsule production, $\mathrm{MEL}$ melanin production, URE urease production, MAT mating, HPX hydrogen peroxide, TBH tert-butyl hydroperoxide, MD menadione, DIA diamide, MMS methyl methanesulphonate, HU hydroxyurea, 5FC 5-flucytosine, AMB amphotericin B, FCZ fluconazole, FDX fludioxonil, TM tunicamycin, DTT dithiothreitol, CDS cadmium sulfate, SDS sodium dodecyl sulfate, CR Congo red, CFW calcofluor white, KCR YPD + 1.5 M KCl, NCR YPD + 1.5 M NaCl, SBR YPD $+2 \mathrm{M}$ sorbitol, KCS YP $+1 \mathrm{M} \mathrm{KCl}$, NCS YP $+1 \mathrm{M} \mathrm{NaCl}$, SBS YP $+2 \mathrm{M}$ sorbitol. 
a
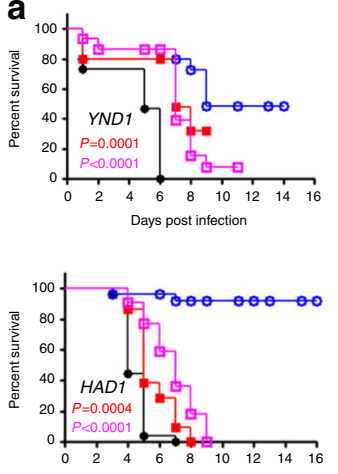

Days post infection
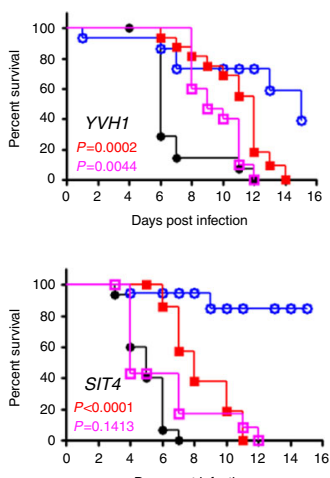

Days post infection
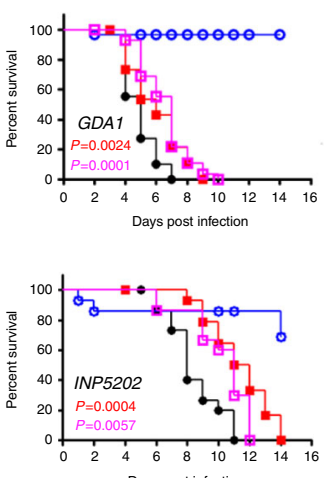

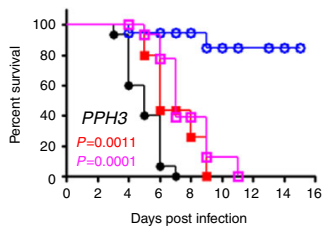

- H99S (WT)

$\vartheta$ PBS

- Mutant 1

$\because$ Mutant

:WT vs

Mutant 1

$P$ value
:WT vs

b

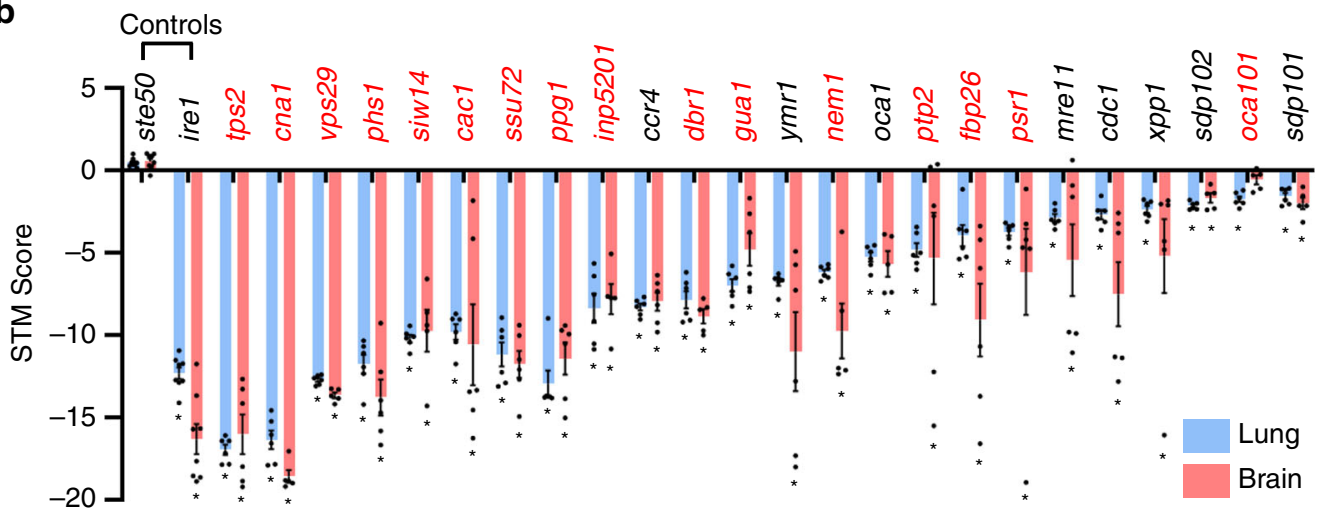

C

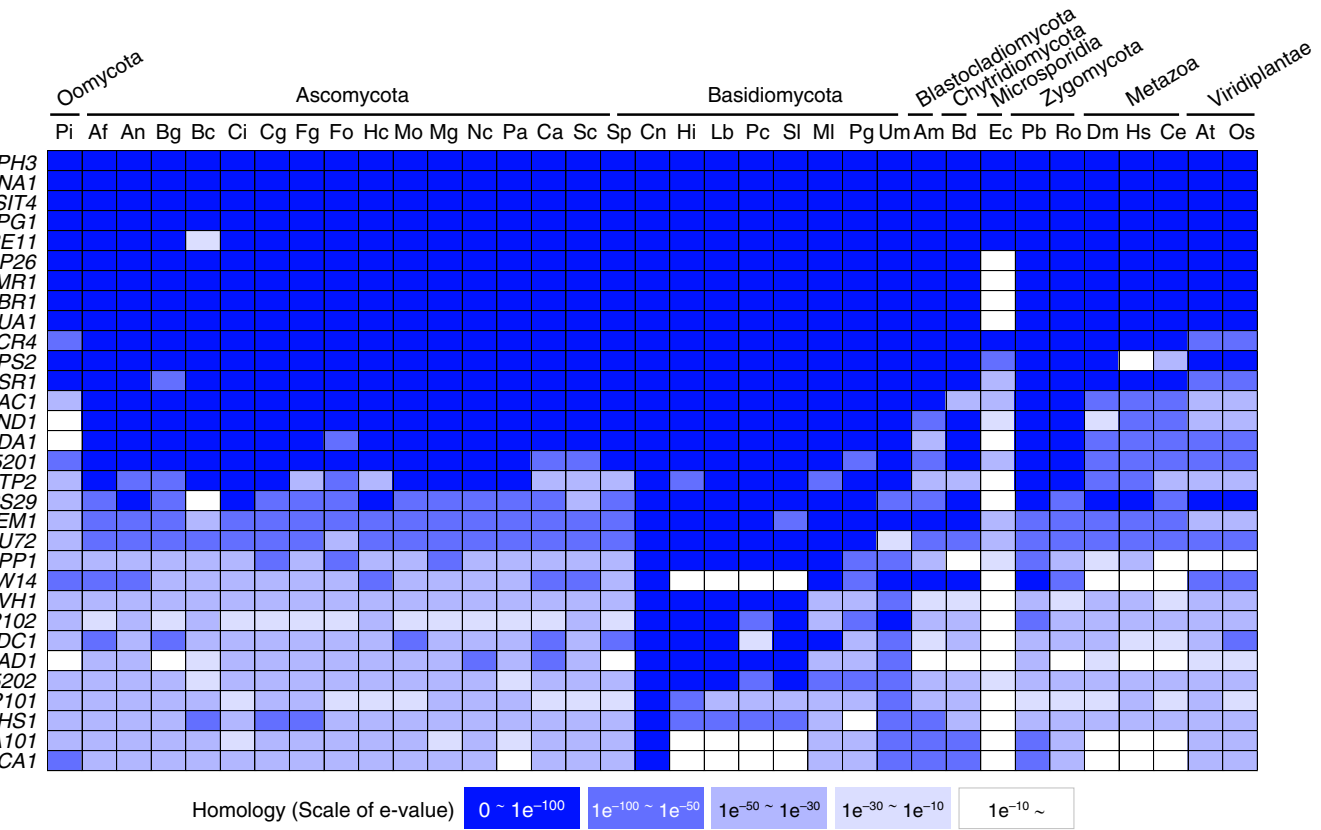

from the lungs to other organs during early infection, phosphatase transcripts can be detected 3-7 dpi.

Phosphatases governing $C$. neoformans pathogenicity. To identify phosphatases required for the pathogenicity of C. neoformans, we performed two large-scale infectivity and virulence assays: (1) a virulence assay using the insect larval model system Galleria mellonella and (2) signature-tagged mutagenesis (STM)based lung and brain infectivity assays using a murine inhalation model. Both methods have been successfully used for identifying virulence-related genes from a large-scale gene-deletion mutant set in previous studies ${ }^{4,5,24}$.

Using the insect-killing virulence assay, we identified 23 phosphatase genes involved in virulence (Fig. $3 \mathrm{a}$ and Supplementary Fig. 4): CNA1, TPS2, CAC1, PTP2, VPS29, PPH3, GDA1, YVH1, SSU72, PHS1, SIW14, DBR1, PSR1, YND1, INP5201, INP5202, HAD1, SIT4, PPG1, GUA1, NEM1, FBP26, and OCA101. STM-based murine lung and brain infectivity revealed 24 infectivity-related phosphatase genes, TPS2, CNA1, VPS29, PHS1, SIW14, CAC1, SSU72, PPG1, INP5201, CCR4, DBR1, GUA1, YMR1, NEM1, OCA1, PTP2, FBP26, PSR1, MRE11, CDC1, 
Fig. 3 Pathogenicity-related phosphatases and their phenotypic traits in C. neoformans. a Virulence-regulating phosphatases were identified by a Galleria mellonella insect killing assay $(n \geq 15)$. P values were calculated using the log-rank (Mantel-Cox) test to measure statistical differences between the WT strain (H99S) and phosphatase mutants. b Infectivity-regulating phosphatases identified by the signature-tagged mutagenesis (STM)-based murine infectivity assay ( $n=6$, summary of Supplementary Fig. 5). STM scores were calculated by quantitative PCR. The ste50 $\Delta$ and ire $\Delta$ mutants were used as virulent positive control and avirulent negative control strains, respectively. The statistically significant was calculated by one-way ANOVA analysis with Bonferroni's multiple comparison test. Data are presented as mean values \pm standard error of mean (SEM). Red letters represent pathogenicity-related phosphatases identified by both murine and insect models. c BLAST matrix comparative search for pathogenicity-related phosphatases was performed using the Comparative Fungal Genomics Platform (http://cfgp.riceblast.snu.ac.kr). Abbreviations: Pi Phytophthora infestans, Af Aspergillus fumigatus, An Aspergillus nidulans, Bg Blumeria graminis, Bc Botrytis cinerea, Ci Coccidioides immitis, Cg Colletotrichum graminicola, Fg Fusarium graminearum, Fo Fusarium oxysporum, Hc Histoplasma capsulatum, Mo Magnaporthe oryzae, Mg Mycosphaerella graminicola, Nc Neurospora crassa, Pa Podospora anserine, Ca Candida albicans, Sc Saccharomyces cerevisiae, Sp Schizosaccharomyces pombe, Cn Cryptococcus neoformans, Hi Heterobasidion irregular, Lb Laccaria bicolour, Pc Phanerochaete chrysosporium, SI Serpula lacrymans, MI Melampsora laricis-populina, Pg Puccinia graminis, Um Ustilago maydis, Am Allomyces macrogynus, Bd Batrachochytrium dendrobatidis, Ec Encephalitozoon cuniculi, Pb Phycomyces blakesleeanus, Ro Rhizopus oryzae, Dm Dorosophila melanogaster, Hs Homo sapiens, Ce Caenorhabditis elegans, At Arabidopsis thaliana, Os Oryza sativa.

XPP1, SDP102, SDP101, and OCA101 (Fig. 3b and Supplementary Fig. 5), 67\% (16/24) of which overlapped with those identified by the insect-killing assay (TPS2, CNA1, VPS29, PHS1, SIW14, CAC1, SSU72, PPG1, INP5201, DBR1, GUA1, NEM1, PTP2, FBP26, PSR1, and OCA101; Fig. 3b, red). Among these, Cna1 (the catalytic subunit of calcineurin) ${ }^{13}$, Cacl (adenylyl cyclase) $)^{11}$, Had1 (haloacid dehalogenase) ${ }^{16}$, and Ptp2 (phosphotyrosine phosphatase $)^{10}$ have previously been reported as requisite for $C$. neoformans virulence, further corroborating our findings.

According to phenome data, 30 out of the 31 pathogenicityrelated phosphatase mutants exhibited at least one phenotypic trait (Fig. 2 and Supplementary Data 7), and a majority showed higher expression in the lungs than other infected tissues (Supplementary Fig. 6). The following in vitro phenotypes were most enriched in $C$. neoformans pathogenicity: membrane integrity $(26 / 31 ; 84 \%)$, DNA damage response $(20 / 31 ; 65 \%)$, melanin production $(18 / 31 ; 58 \%)$, cell wall integrity $(13 / 31 ; 42 \%)$, and growth at $37^{\circ} \mathrm{C}(14 / 31 ; 45 \%)$ (Fig. 2). In contrast, the $s d p 101 \Delta$ mutant did not exhibit an in vitro phenotype. Notably, deletion of Sdp102, a dual-specificity MAPK phosphatase paralogous to Sdp101, resulted in minor phenotypic alteration and reduced infectivity (Figs. 2 and $3 b$ ), suggesting that Sdp101 and Sdp102 may play redundant roles in the pathogenicity of $C$. neoformans. To identify the functional correlation between Sdp101 and Sdp102, we tried to construct the $s d p 101 \Delta s d p 102 \Delta$ double mutant strains but failed, even after repeated attempts (data not shown). Similarly, although Inp5201 is paralogous to

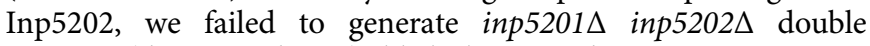
mutants (data not shown), likely because the inp5201 $\Delta$ mutant alone showed severe growth defects even at $30^{\circ} \mathrm{C}$ (Figs. 2 and $4 \mathrm{a}$ ). Therefore, Sdp101/Sp102 and Inp5201/Inp5202 may have a synthetic lethal relationship. Conversely, although Ocal and Oca101 are also paralogous, we successfully obtained an oca1 $\Delta$ oca101s double mutant, but we did not find any additive or synergistic phenotypic traits in the oca $1 \Delta$ oca $101 \Delta$ double mutant compared to either single mutant (Supplementary Fig. 7), suggesting that Ocal and Oca101 may independently contribute to the pathogenicity of $C$. neoformans.

Of the 31 pathogenicity-related phosphatases identified, five do not have evident orthologues in humans (Hs in Fig. 3c): Tps2, Siw14, Had1, Oca101, and Oca1. Therefore, these five pathogenicity-related phosphatases could be excellent anticryptococcal targets. Tps2, Had1, and Oca1 are also required for the virulence of $C$. albicans ${ }^{25-27}$, thus drugs that target these phosphatases could have broad antifungal activity.

Phosphatases involved in growth at mammalian body temperature. We next focused on the pathobiological functions of the 31 pathogenicity-related phosphatases in C. neoformans. First, because thermotolerance for mammalian body temperatures is a critical virulence factor for most human fungal pathogens, we quantitatively measured growth of each mutant at 30 and $37^{\circ} \mathrm{C}$. The gua $1 \Delta$, yvh $1 \Delta, f b p 26 \Delta$, siw $14 \Delta, d b r 1 \Delta, \operatorname{ccr} 4 \Delta, p p g 1 \Delta$, nem $1 \Delta$, and inp5201 $\Delta$ mutants showed impaired growth at both 30 and $37^{\circ} \mathrm{C}$ (Fig. 4a and Supplementary Fig. 8). Of these, the ccr $4 \Delta$, $\operatorname{ppg} 1 \Delta$, nem $1 \Delta, d b r 1 \Delta$, and inp5201s mutants exhibited more growth defects at $37^{\circ} \mathrm{C}$ than $30^{\circ} \mathrm{C}$. The ssu $72 \Delta$, phs $1 \Delta$, mre $11 \Delta$, tps $2 \Delta$, and cna $1 \Delta$ mutants showed impaired growth at $37^{\circ} \mathrm{C}$ but not at $30^{\circ} \mathrm{C}$. A total of 14 phosphatase mutants showed impaired growth at $37^{\circ} \mathrm{C}$ relative to WT (Fig. 4a and Supplementary Fig. 8) and showed reduced murine infectivity or insect virulence relative

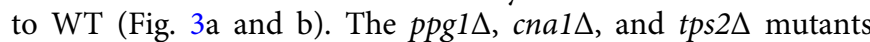
exhibited the most significant growth defects at $37^{\circ} \mathrm{C}$ and did not grow to the level of the WT even after an extended incubation period (Fig. 4a). Concordantly, $p p g 1 \Delta$, cna $1 \Delta$, and tps $2 \Delta$ mutants exhibited highly reduced lung and brain STM values $(<-5$; Fig. 3b). The oca $101 \Delta$ mutant showed impaired growth relative to WT at $30^{\circ} \mathrm{C}$ but not at $37^{\circ} \mathrm{C}$ (Supplementary Fig. 8) suggesting that the role of Ocal01 in C. neoformans pathogenicity is not related to temperature. Collectively, these data suggest that growth at $37^{\circ} \mathrm{C}$ is a critical for virulence of C. neoformans.

Phosphatases involved in melanin and capsule production. C. neoformans has two major virulence factors: the polyphenol pigment melanin and the polysaccharide capsule, both of which contribute to its antiphagocytic activity ${ }^{28,29}$. The melanin pigment also serves as antioxidant due to its reactive oxygen species scavenging activity ${ }^{28}$. Among 19 phosphatase mutants defective in melanin production on Niger seed medium, the following 13 mutants also exhibited defective melanin production on L-DOPA and epinephrine media (Fig. 4b): mre11 $, c c r 4 \Delta, v p s 29 \Delta, y v h 1 \Delta$, $f b p 26 \Delta$, inp5201 $, c a c 1 \Delta, p t p 2 \Delta, p t c 2 \Delta, d b r 1 \Delta, p p g 1 \Delta$, nem $1 \Delta$, and gua $1 \Delta$. All of these mutants, except the ptc2 $\Delta$ mutant, exhibited reduced infectivity or virulence (Fig. 3a and b), suggesting that melanin production is strongly correlated with pathogenicity. In contrast, SIW14 deletion increased melanin production in all melanin-inducing media (Fig. 4b) but attenuated the virulence of $C$. neoformans (Fig. 3b), suggesting that other cellular functions mediated by Siw14 may promote virulence.

We next addressed whether these phosphatases are directly involved in induction of $L A C 1$, which encodes laccase, a ratelimiting enzyme for melanin production in C. neoformans, under nutrient-starvation conditions ${ }^{30}$ (Fig. 4c). Deletion of PTP2, CCR4, INP5201, CAC1, DBR1, FBP26, GUA1, NEM1, and PPG1 significantly reduced $L A C 1$ induction upon nutrient starvation (Fig. 4c), with deletion of PTP2, CCR4, CAC1, and PPG1 almost completely abolishing $L A C 1$ induction. Ptp2 has been reported as 

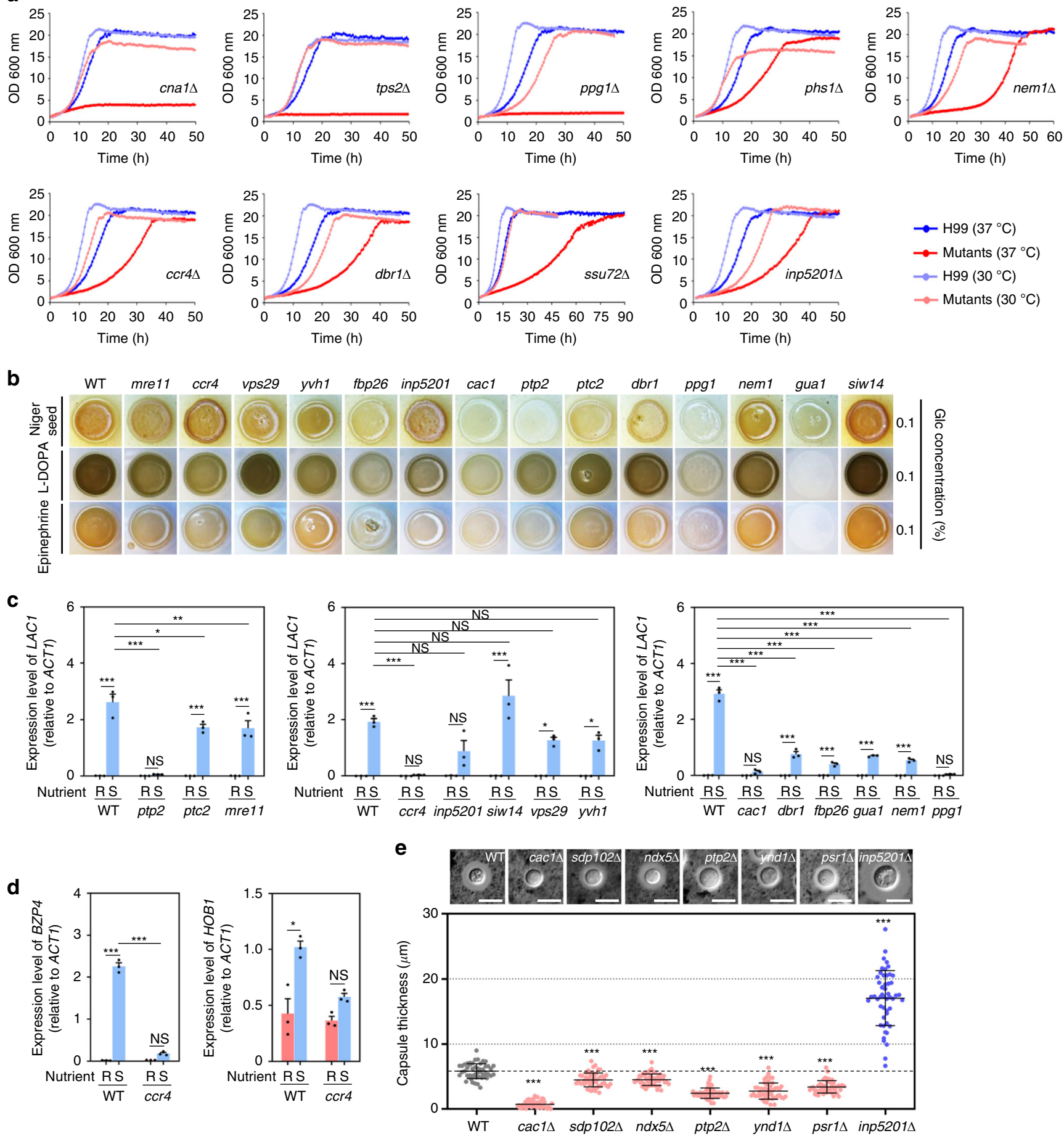

a negative feedback regulator of the Hog1 MAPK; Hog1 deletion increases $L A C 1$ induction and melanin production ${ }^{10,30}$. Recently, we have reported that $L A C 1$ induction is controlled by the following four core TFs: Bzp4, Usv101, Hob1, and Mbs130. Among these, expression of BZP4 and HOB1 is induced by nutrient starvation, and BZP4 induction itself is governed by Hob $1^{30}$. Therefore, we examined whether the LAC1-regulating phosphatases also regulate induction of BZP4 and $H O B 1$ (Fig. $4 \mathrm{~d}$ and Supplementary Fig. 9). Although BZP4 induction by nutrient starvation was significantly reduced in most melanin-defective phosphatase mutants, CCR4 deletion in particular abolished BZP4 (Fig. 4d) and, notably, HOB1 induction upon nutrient starvation (Fig. 4d). Therefore, the Ccr4-Hob1-Bzp4-dependent signalling pathway appears to be critical for $L A C 1$ induction and melanin production in C. neoformans.

We next focused on phosphatases involved in capsule production. When capsule production was quantitatively measured as packed cell volume, Cac1, Ptp2, Psr1, Ndx5, Hpp2, Oca1, Sdp102, Ynd1, Ngl3, Ppp5, and Cwh43 positively impacted cell volume, and Inp5201, Dbr1, Phs1, Cdc1, Cna1, Sit4, Ccr4, Nem1, Pcd102, and Fig4 negatively impacted it (Supplementary Fig. 10). For these mutants, we examined actual capsule thickness; among these, six mutants $(c a c 1 \Delta, s d p 102 \Delta, n d x 5 \Delta$, ptp2 $2 \Delta, y n d 1 \Delta$, and psr1 $1 \Delta$ ) showed reduced capsule production (Fig. $4 \mathrm{e}$ and Supplementary Fig. 10) that mirrored their phenotype in cell volume assays, and five of these $(\operatorname{cac} 1 \Delta, p t p 2 \Delta, s d p 102 \Delta, y n d 1 \Delta$, 
Fig. 4 Phosphatases involved in major virulence traits of $\mathbf{C}$. neoformans. a Growth curves of WT and phosphatase mutants were generated at $30^{\circ} \mathrm{C}$ (control) and $37^{\circ} \mathrm{C}$ (mammalian body temperature). Fifteen phosphatase mutants had growth defects at 30 and $37^{\circ} \mathrm{C}$. Nine of these phosphatase mutants had more substantial growth defects at $37^{\circ} \mathrm{C}$ than $30^{\circ} \mathrm{C}$ (additional data in Supplementary Fig. 8). Each curve represents data from two independent experiments (see Supplementary Fig. 8 for data from the individual experiments). Optical density at $600 \mathrm{~nm}\left(\mathrm{OD}_{600 \mathrm{~nm}}\right)$ was measured with a multichannel bioreactor (Biosan Laboratories, Inc., Warren, MI, USA) for 40-90 h based on growth rate. b Melanin production was measured using three different melanin-inducing media (Niger seed, dopamine, and epinephrine medium). Representative images from three independent experiments are shown here. Each strain was spotted on medium containing $0.1 \%$ glucose, incubated at $30^{\circ} \mathrm{C}$, and photographed after $1-3$ days. c, $\mathbf{d}$ Gene expression of the melanin-regulating genes $L A C 1, B Z P 4$, and HOB1 were determined by qRT-PCR in both nutrient-rich (R) and nutrient-starvation (S) conditions. RNA was extracted from three biological replicates with three technical replicates of WT and melanin-regulating phosphatase mutants. Expression was normalized to $A C T 1$, and statistical significance was calculated by one-way ANOVA analysis with Bonferroni's multiple comparison test. Data are presented as mean values \pm SEM ( $\left.{ }^{\star} P<0.05,{ }^{\star \star} P<0.001,{ }^{\star \star \star} P<0.0001\right)$. e The capsule production assay was performed using capsule-inducing media (FBS agar medium). Capsule thickness (total diameter-cell body diameter) was measured for WT cells $(n=50)$ and for each phosphatase mutant $(n=50)$. Statistical significance was calculated by one-way ANOVA analysis with Bonferroni's multiple comparison test. Data are presented as mean values \pm SEM ( ${ }^{*} P<0.05$, $\left.{ }^{\star \star} P<0.001,{ }^{\star \star \star} P<0.0001\right)$. The graph is representative of more than three independent experiments. The images are representative DIC images of WT and phosphatase mutants incubated on FBS agar medium and stained with India ink. Scale bars, $10 \mu \mathrm{m}$.

psr1 $1 \Delta$ ) also showed reduced virulence (Fig. 3). In contrast, the inp5201s mutant again showed highly enhanced capsule production but exhibited dramatically reduced virulence (STM $<-7$ ), the latter of which likely resulted from defective melanin production (Fig. 4b). Overall, the ability to produce melanin and the polysaccharide capsule were highly correlated with pathogenic potential in C. neoformans.

The retromer complex promotes $C$. neoformans virulence. Among the newly identified virulence-related phosphatases in this study, Vps29 (CNAG_00182) is a putative component of the retromer complex, first discovered in $S$. cerevisiae ${ }^{31}$. The yeast retromer is a cytosolic, heteropentameric protein complex that mediates the intracellular trafficking of protein cargo from the post-Golgi organelles to the lytic compartment ${ }^{32,33}$. It consists of the cargo-recognition core (CRC) complex, which contains Vps29, Vps35, and Vps26, and a membrane-deforming sorting nexin (SNX) complex, which contains Vps5 and $\mathrm{Vps} 17^{32}$.

To address whether functions of Vps29 resulted from the conserved role of the retromer complex, we functionally characterized other retromer components in C. neoformans. In the $C$. neoformans genome, we identified all of the remaining retromer component genes encoding proteins orthologous to Vps35 (CNAG_01837), Vps26 (CNAG_01426), Vps5 (CNAG_01315), and Vps17 (CNAG_00508), suggesting that the retromer complex is evolutionarily conserved in this fungal pathogen. We deleted each gene in the H99 strain and performed phenotypic analyses (Supplementary Figs. 11 and 12). Notably, deletion of VPS35 and VPS26 resulted in more dramatic phenotypic changes than that of VPS29 (Fig. 5a), indicating that Vps35 and Vps26 are critical CRC complex components in $C$. neoformans. In contrast, deletion of SNX components VPS5 and VPS17 resulted in relatively minor phenotypic changes (Fig. 5a). Supporting this finding, CRC complex mutants showed significantly reduced virulence in the insect-killing assay whereas SNX complex mutants remained as virulent as the wild-type strain (Fig. 5b). However, both CRC and SNX complex mutants exhibited markedly reduced lung and brain infectivity in a murine-based STM analysis (Fig. 5c). Collectively, these findings indicate that the retromer CRC and SNX complexes are critical for the pathogenicity of C. neoformans.

Gda1 and Ynd1 modulate $O$-mannosylation in $C$. neoformans. In $S$. cerevisiae, Gdal and Ynd1 are Golgi membrane-bound apyrases that modulate mannosylation of $\mathrm{O}$-linked and $\mathrm{N}$-linked glycoproteins and glycosphingolipids by affecting the antiport exchange ratio between GDP-mannose and $\mathrm{GMP}^{34,35}$. Gda1 exhibits activity highly specific to GDP whereas Ynd1 shows a much broader spectrum of activity ${ }^{35,36}$. In accord, double deletion of GDA1 and YND1 results in more severe glycosylation defects than deletion of either gene alone ${ }^{35}$. To investigate whether $C$. neoformans gda $1 \Delta$ and $y n d 1 \Delta$ mutants show defects in $O$ glycosylation, as observed in S. cerevisiae and C. albicans mutant strains $^{35,37}$, we compared the $O$-glycan profiles of wild-type, $g d a 1 \Delta$, and $y n d 1 \Delta$ strains. The O-linked oligosaccharides assembled on cell wall mannoproteins (cwMPs) of C. neoformans cells were obtained by hydrazinolysis, labelled with 2aminobenzoic acid (2-AA), and then analysed using HPLC with fluorescence detection (Fig. 6a). Consistent with a previous report $^{38}$, O-linked oligosaccharides from the C. neoformans wildtype strain were mostly composed of 2-4 mannose residues (Man2-Man4; M2-M4), with minor O-glycan species containing xylose (X1M2-X1M4) and a minor a1,2-mannotriose (M3*) species (Fig. 6a). In the O-glycan profile of the gda1 1 mutants, the M4 peak was dramatically reduced, and the M1 peak was increased compared to those in the wild-type strain (Fig. 6b). Notably, the minor xylose-containing species became more detectable in the O-glycans of the gda1s strain (Fig. 6b). The ynd1 $\Delta$ mutants also showed a significantly decreased M4 peak with the increased M1 peak, although the M4 peak decreased to a lesser degree than that of the gda1 $\Delta$ mutants (Fig. 6b). These altered profiles strongly indicate a severe defect in the elongation process of $O$-glycans, which is likely due to inefficient GDPmannose supply from the cytosol to the lumen of the Golgi caused by decreased GDPase activity in the gda1 $\Delta$ and $y$ nd $1 \Delta$ strains (Fig. 6c). Thus, the results collectively suggest that GDA1 and YND1 in C. neoformans encode membrane-bound apyrases required for Golgi $N$ - and $O$-glycosylation (Fig. 6c), consistent with these genes' function in other yeasts. The more dramatic reduction of major $O$-glycans in $g d a 1 \Delta$ than $y n d 1 \Delta$ indicates that Gdalp is a major GDPase responsible for GDP-mannose supply to the major $O$-glycan biosynthesis pathway. The reduced capsule size in $y n d 1 \Delta$ (Fig. 4e) suggests that Ynd1p might be also involved in the GDP-mannose supply to capsule biosynthesis. Notably, however, we failed to obtain gda1s ynd $1 \Delta$ double mutants (data not shown), indicating that the two proteins may have a synthetic lethal relationship in $C$. neoformans. Because both gda $1 \Delta$ and ynd $1 \Delta$ mutants exhibited reduced virulence in the insect killing assay (Fig. 3a), these results collectively suggest that $O$-mannosylation is critical for virulence of C. neoformans.

Phosphatases involved in the blood-brain barrier (BBB) crossing. The most lethal damage conferred by C. neoformans is brain infection, which generally results in fatal meningoencephalitis. Notably, the brain STM scores for all of the phosphatase mutants 
a

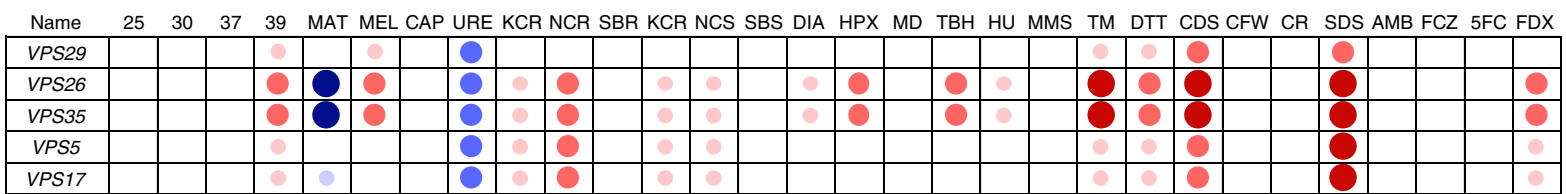

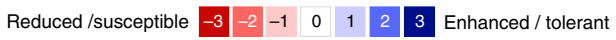
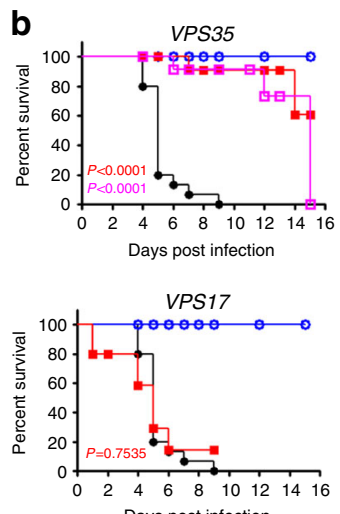

Days post infection
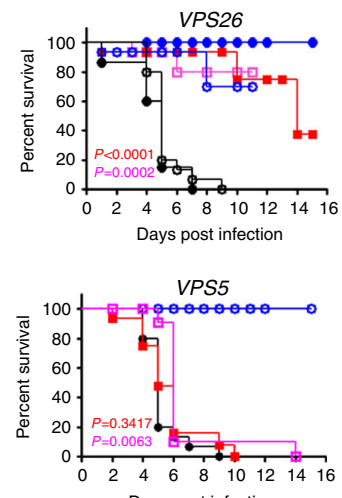

Days post infection

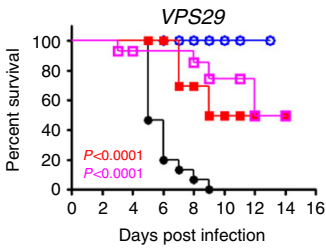

$P$ value
Hogs (WT)

$\begin{array}{lll}- \text { H99S (WT) } & \text { :WT vs } \\ \text { Mutant } 1 & \text { PBS }\end{array}$

- Mutant 1

Pvalue
$:$ WT vs

Mutant 2

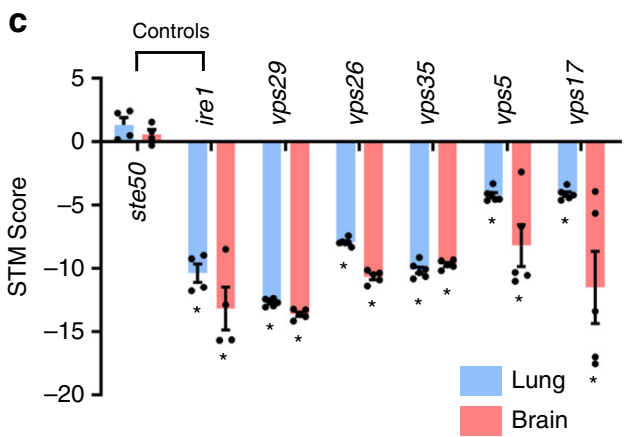

Fig. 5 The role of retromer complex in the pathogenicity of $\mathbf{C}$. neoformans. a Phenotypic heatmap of retromer complex mutants constructed based on

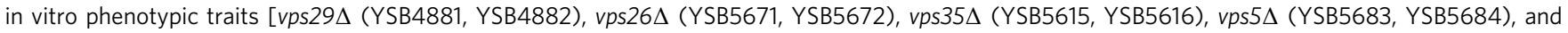
vps17 $\Delta$ (YSB5724)] (Supplementary Fig. 12). b, c The retromer complex is involved in the pathogenicity of $C$. neoformans. $\mathbf{b}$ Insect-killing assay performed for the retromer complex mutants $(n \geq 15)$. $P$ values shown in the graph were calculated using the log-rank (Mantel-Cox) test to measure statistical differences between the wild-type (WT) strain (H99S) and each phosphatase mutant strain. In the VPS26 graph, filled and empty circles indicate two independent PBS and WT-infection controls. c Signature-tagged mutagenesis (STM)-based murine infectivity assay $(n=3)$. STM scores were calculated by quantitative PCR. The ste50 $\Delta$ and ire $\Delta$ mutants were used as virulent-positive control and avirulent-negative control strains, respectively. The statistically significant was calculated by one-way ANOVA analysis with Bonferroni's multiple comparison test. Data are presented as mean values \pm SEM $\left({ }^{\star} P<0.05\right)$.

were generally similar to their lung STM scores, although some of them exhibited lower brain scores (Fig. 3b; TPS2, YMR1, FBP26, MRE11, CDC1, and XPP1). However, because the phosphatase mutants administered through intranasal inhalation pass through the lungs first, those reaching the brain were less equally distributed than in pooled input mutants, which made direct comparison of lung and brain STM scores difficult. Therefore, to further address the role of these phosphatases in brain infection, we monitored the ability of the pathogenicity-related phosphatase mutants, except those showing reduced growth at $37^{\circ} \mathrm{C}$, to traverse the $\mathrm{BBB}$. Among these, five mutants $(x p p 1 \Delta, s s u 72 \Delta$, siw $14 \Delta$, sit $4 \Delta$, and gda1 $\Delta$ ) showed significantly reduced ability to traverse the BBB (Fig. 7a). We recently showed that adhesion to the $\mathrm{BBB}$ is a prerequisite for effective $\mathrm{BBB}$ crossing ${ }^{39}$, and, indeed, $x p p 1 \Delta$, ssu $72 \Delta$, siw $14 \Delta$, and sit4 $\Delta$ mutants, but not gda1 $\Delta$, showed reduced BBB adhesion (Fig. $7 \mathrm{~b}$ ), suggesting that reduced $O$-mannosylation of proteins or lipids is required for $\mathrm{BBB}$ crossing but not adhesion. We found that $C$. neoformans does not actively grow at $37^{\circ} \mathrm{C}$ in the tissue culture medium that we used for the $24 \mathrm{~h}$ in vitro BBB crossing and adhesion assays (data not shown), probably due to the low glucose concentration ( $0.1 \%$ glucose). Therefore, it is unlikely that Cryptococcus replication in the bottom well complicated our data.

We recently reported several TFs that promote BBB adhesion that are highly induced in vitro by host-mimic conditions (HMC; RPMI medium containing $10 \% \mathrm{FBS}$ at $37^{\circ} \mathrm{C}$ under $5 \% \mathrm{CO}_{2}$ ): PDR802, FZC31, and GAT201 ${ }^{39}$. Therefore, we next addressed whether these genes, in addition to known brain infection-related genes [inositol transporter genes (ITR1a and ITR3c) and MPR1]

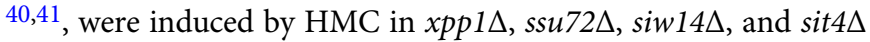
mutants. Among these, SSU72 deletion most markedly reduced HMC-mediated MPR1 induction (Fig. 7c), suggesting that the defects of ssu $72 \Delta$ mutants in $B B B$ crossing and adhesion are at least partially caused by reduced $M P R 1$ induction. However, because the ssu $72 \Delta$ mutants were more defective than the mpr1s mutants in BBB crossing and adhesion (Fig. 7a and b), other cellular functions of Ssu72 may also be involved in BBB crossing and adhesion. Deletion of SIT4 and GDA1 markedly reduced HMC-mediated ITR $3 c$ induction (Fig. 7c). GDA1 deletion also affected FZC31 induction (Fig. 7c). However, induction of GAT201 and PDR802 was not significantly affected by deletion of SIT4, SIW14, SSU72, GDA1, or XPP1 (Fig. 7c).

To obtain insight into the integrated signalling networks governing brain infection by $C$. neoformans, we generated functional gene networks using STRING analysis by combining data from this and previously published studies on BBB-crossingrelated phosphatases, TFs, and kinases (Fig. $7 \mathrm{~d})^{39}$. We found that genes involved in glucose sensing (Sit4-Snf1-Gal83), RNA processing (Ssu72), and purine metabolism (Xpp1-Met3) were critical for BBB crossing by C. neoformans. Collectively, these findings indicate that $C$. neoformans utilizes complex signalling networks for brain infection.

Comparison of fungal pathogenicity-related phosphatases. By comparing the virulence data of phosphatase mutants in $C$. neoformans with those available in other plant and animal pathogenic fungi ${ }^{8,25-27,42-65}$, we found several core fungal pathogenicity-related phosphatases (Fig. 8 and Supplementary Data 9). In the human yeast pathogens $C$. neoformans and $C$. albicans, the following 13 phosphatases are considered core pathogenicity-related phosphatases: Cnal/Cmp1, Sit4, Oca1, Yvh1, Sdp101/Cpp1, Ptp2/Ptp3, Cac1/Cyr1, Ccr4, Had1/Rhr2, Tps2, Inp5201/Inp51, Ppg1, and Gua1. Of the 13 phosphatases, CnaA, SitA, and OrlA (a Tps2 ortholog) have been shown to be required for $A$. fumigatus virulence ${ }^{53-55}$. Upon comparison with pathogenicity-related phosphatases in F. graminearum, eight 
a

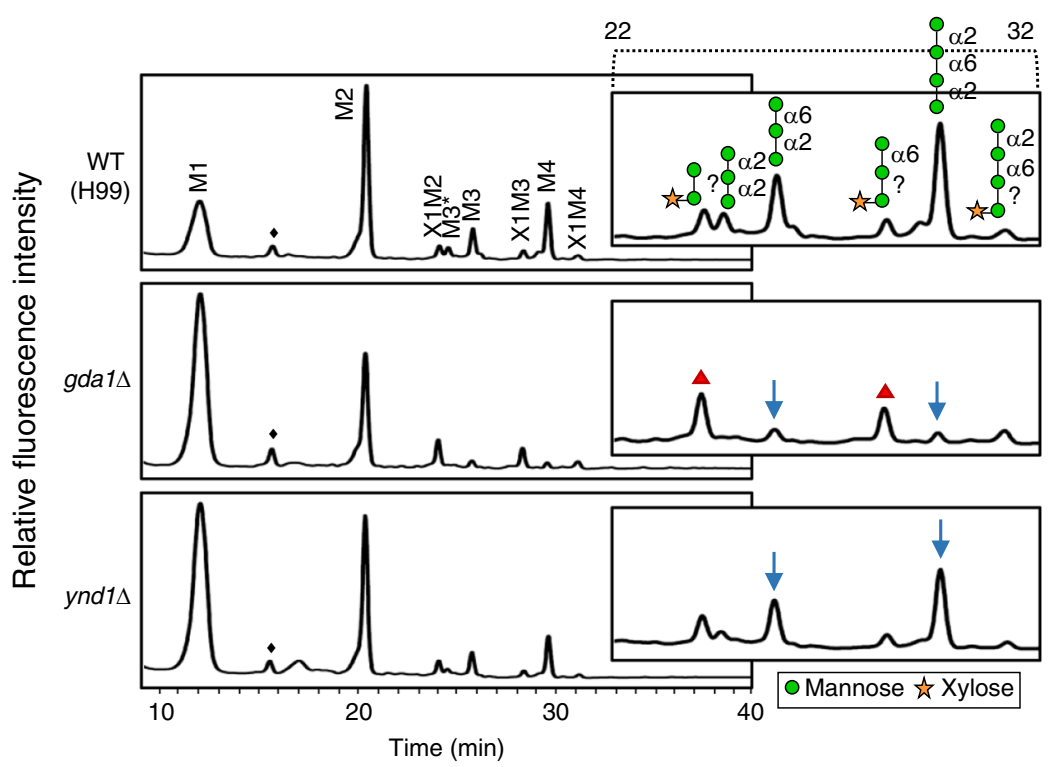

b
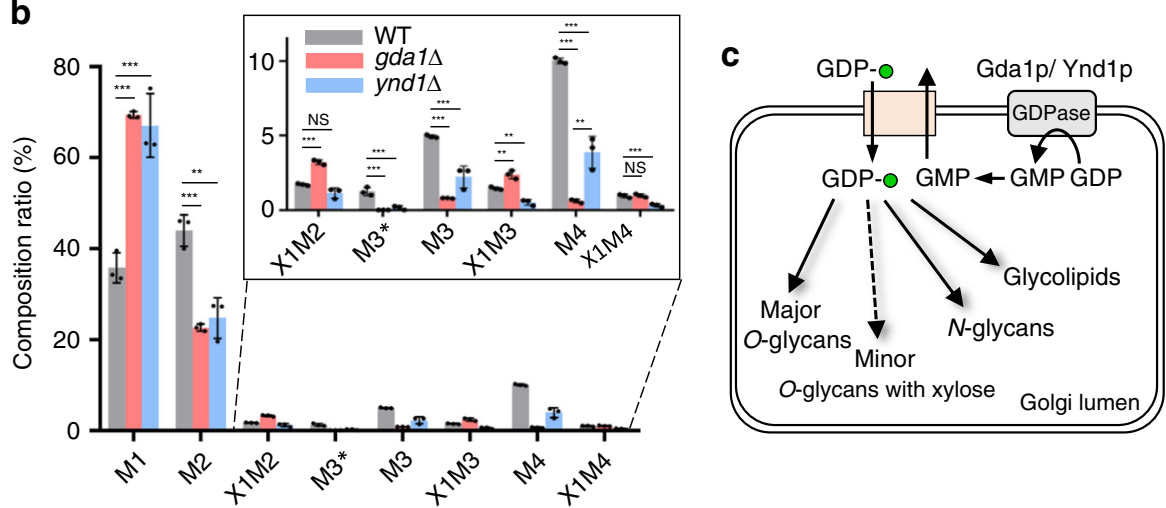

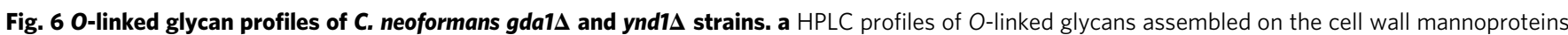
of C. neoformans wild-type (WT), gda1s (YSB4750), and ynd1 (YSB4856) strains. To distinguish small peaks more clearly, HPLC profiles from 22 to 32 $\min$ in the $x$ - and $y$-axes were enlarged and inserted. diamond, unknown peak. M mannose, $X$ xylose, M3* $\alpha 1,2$-mannotriose. Arrow, decreased M3 and M4 peak; triangle, increased $\mathrm{O}$-glycan species containing xylose (X1M2-X1M4) in the mutant strains. $\mathbf{b} \mathrm{O}$-glycan composition of each $\mathrm{C}$. neoformans strain. For quantitative analysis, the area of each peak in the HPLC profile from three independent experiments was measured and presented as percentage of the total area of all peaks. Statistical significance was calculated by one-way ANOVA analysis with Bonferroni's multiple comparison test. Data are presented as mean values \pm standard deviation (SD) $\left({ }^{\star} P<0.05 ;{ }^{* \star} P<0.001 ;{ }^{\star \star \star} P<0.0001\right)$. c The proposed function of Gda1p and Ynd1p to provide donor substrates for mannosylation of proteins and lipids in the Golgi apparatus of $C$. neoformans.

phosphatases have been shown to be required for the virulence of both animal and plant fungal pathogens: Sit4, Yvh1, Sdp2/Msg5 (an Sdp101 orthologue), Ptp2, Ac1 (a Cac1 orthologue), Tps2, Inp53 (an Inp5201 orthologue), and Ppg1. Sit4 is involved in the TOR pathway, Cacl is involved in the cAMP pathway, and Ppg1 and Yvh1 $1^{51,66-68}$ are involved in cell growth, nutrient sensing, and the stress response in fungal pathogens ${ }^{2,69}$. The Cnal and Had1-mediated calcineurin pathway, the Tps2-mediated trehalose pathway, and the Msg5-mediated Mpk1/Slt2 MAPK pathway are all required for maintaining cell wall integrity. Ptp2, which is a major negative feedback regulator of the HOG pathway, is involved in adaptation and the stress response in fungal pathogens. Inp51, Inp52, and Inp53 are involved in phosphoinositide signalling, which controls vesicle trafficking, the actin cytoskeleton, and cell wall integrity ${ }^{70,71}$. Based on these data, phosphatases and signalling pathways involved in cell growth, nutrient sensing, cell wall integrity, the stress response, and phosphoinositide signalling appear to play pivotal roles in general fungal pathogenicity. Notably, however, deletion of $P P H 3$ reduces $C$. neoformans and F. graminearum virulence but enhances $C$. albicans virulence ${ }^{8,42}$. In contrast, deletion of PTC2 and PTC3 reduces $C$. albicans and F. graminearum virulence, respectively, but does not reduce $C$. neoformans virulence ${ }^{27,72}$. Thus, some phosphatases may play differential roles in controlling the virulence of various fungal pathogens.

\section{Discussion}

In this study, we identified a total of 139 phosphatases in $C$. neoformans, including protein phosphatases, lipid/nucleoside/carbohydrate phosphatases, and pyrophosphatases, and constructed a high-quality library of 219 signature-tagged gene-deletion mutant strains representing 109 phosphatases for functional analysis. By incorporating 11 additional signature-tagged mutants representing Six phosphatases that we previously constructed $4,5,10,11$, we systematically analysed the in vitro and in vivo phenotypic traits of a total of 230 signature-tagged mutant strains representing 114 phosphatases ( $82 \%$ of original 139). Under 30 distinct in vitro growth conditions (e.g., temperature-dependent growth, mating, 
a

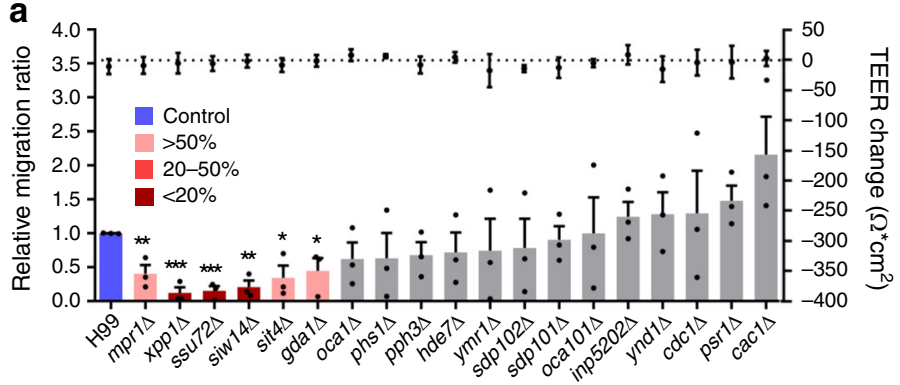

b

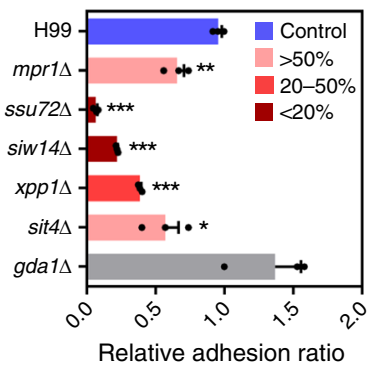

C

C $\quad$ Basal $\amalg$ h in RPMI + FBS

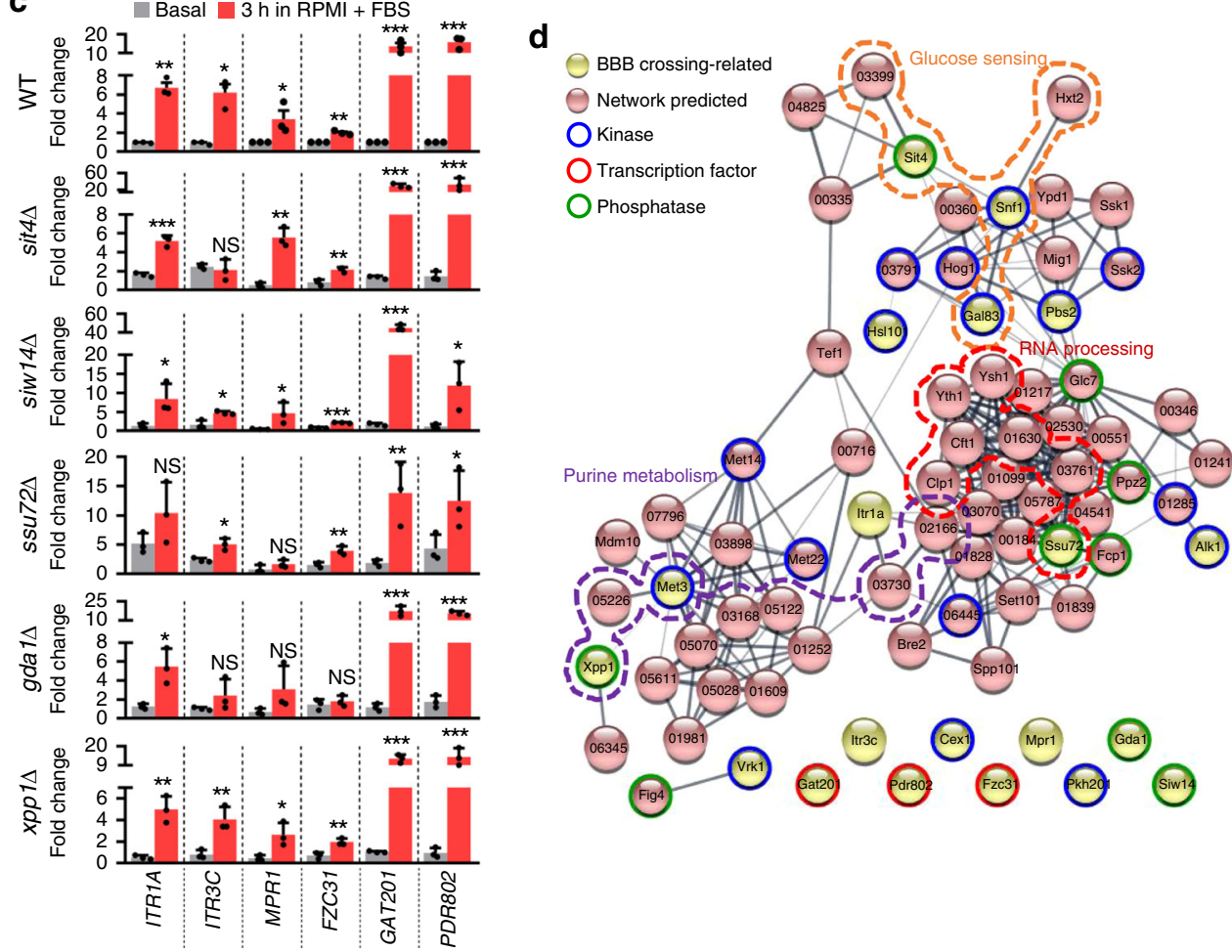

Fig. 7 In vitro BBB transmigration and adhesion assays for $\mathbf{C}$. neoformans phosphatases. a In vitro BBB migration and $\mathbf{b}$ human brain microvascular endothelial cell line (hCMEC/D3) adhesion assay of pathogenicity-related phosphatases. Right: $y$-axis indicates trans-endothelial electrical resistance (TEER). Data plots represent individual data from three independent experiments $(n=3)$. Data presented as mean values \pm SEM. Significant differences were calculated by two-tailed (unpaired) Student's t-test comparing wild-type (WT) and each phosphatase deletion mutant. c Host-mimic condition (HMC)-mediated induction of brain infection-related genes in wild-type (WT) and phosphatase deletion mutant strains. Gene expression was determined by quantitative RT-PCR with CDNA synthesized from total RNA prepared from cells shifted from basal condition (YPD at $30^{\circ} \mathrm{C}$, grey shaded) to $\mathrm{HMC}$ (RPMI with $10 \%$ foetal bovine serum at $37^{\circ} \mathrm{C}$ under $5 \% \mathrm{CO}_{2}$ ) and further incubated for $3 \mathrm{~h}$ (red shaded). Fold-change of gene expression was calculated relative to basal expression levels of each gene in WT. Data from three independent experiments (black dots) are presented as mean values \pm SEM. Statistical significance between basal and HMC was determined by two-tailed (unpaired) Student's $t$-test $\left({ }^{\star} P<0.05 ;{ }^{* \star} P<0.001 ;{ }^{\star \star \star} P<0.0001\right)$. d Functional protein association network analysis of BBB crossing-related TFs, kinases, and phosphatases of C. neoformans predicted by STRING (http:// string-db.org) with protein sequences obtained from FungiDB (https://fungidb.org/fungidb/). Images were drawn with Cytoscape v3.7.2. Dotted lines: groups of genes involved in purine metabolism (purple), RNA processing (red), and glucose sensing (orange).

capsule/melanin/urease production, stress and antifungal drug resistance), 60 out of 114 mutants (53\%) exhibited at least one phenotypic trait. Among the remaining 54 mutants that did not show a phenotypic trait under these conditions, none possessed infectivity and virulence defects, suggesting that these 54 phosphatases may not be involved in the pathobiology of C. neoformans. Among these, however, functionally redundant phosphatases could exist, and evident phenotypes may only result from their combined mutation. In particular, our in vivo expression profiling revealed that the following phosphatase genes were markedly upregulated during host infection: CDC1, GDA1, GEP4, NDX2, NDX3, PTP3, APH4, HAD9, YMR1, INM1, PPU1, DPP101, and AKP1 (Supplementary Data 8). Therefore, we recommend that the role of these phosphatases be further characterized in future work.

To identify functional correlations between TFs, kinases, and phosphatases, we attempted to make co-phenotypic clusters of TF, kinase, and phosphatase mutants constructed by this and previous studies 4,5 , but found that even well-established correlations between known signalling components, such as Hog1 and Ptp2 and Cna1 and Crz1, were not evident in the co-clustering analysis. There could be several explanations for this. Mutation of a phosphatase gene, which functions as a negative feedback regulator of a kinase-dependent pathway, may not lead to a 
a

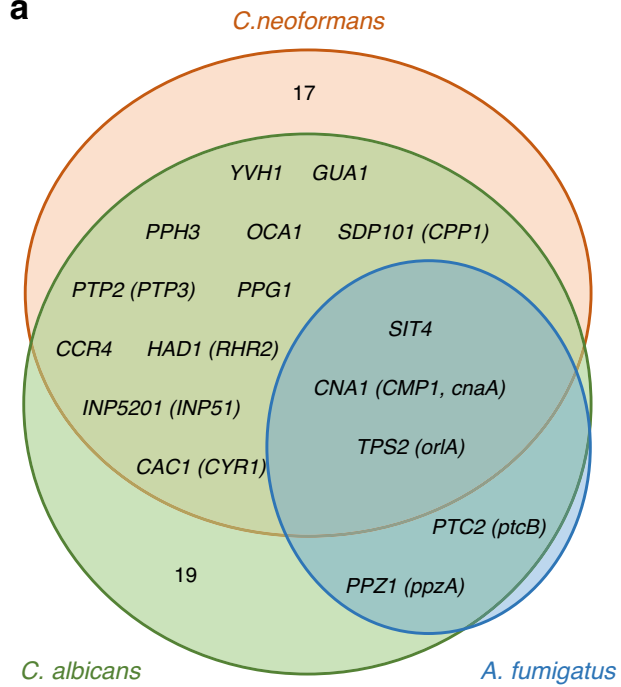

b

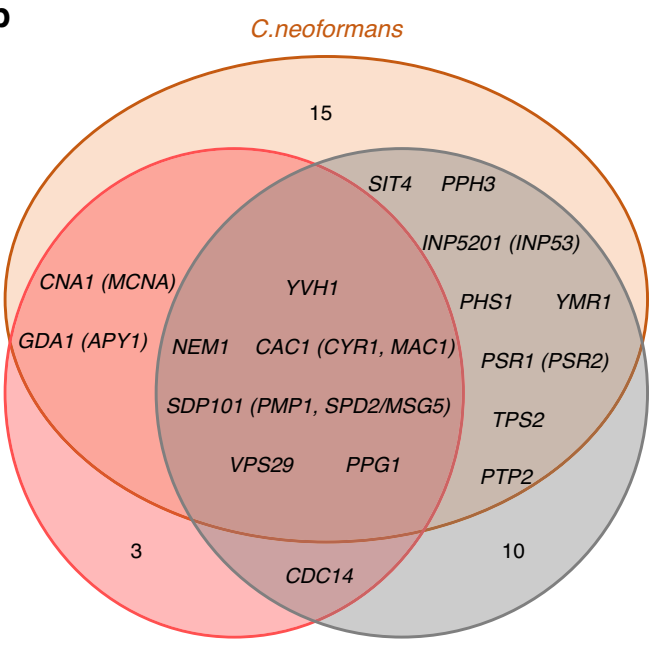

M. oryzae

F. graminearum

Fig. 8 Pathogenicity-related phosphatases in fungal pathogens. a Venn diagram showing the distribution of pathogenicity-related phosphatases in the human fungal pathogens C. neoformans, Candida albicans, and Aspergillus fumigatus. b Venn diagram showing the distribution of pathogenicity-related phosphatases in C. neoformans and plant-pathogenic filamentous fungi Fusarium graminearum and Magnaporthe oryzae.

phenotype opposite to the kinase mutant phenotype. For example, mutations in PTP2, which encodes a major negative feedback regulator of Hog1 MAPK, results in phenotypes similar to hog1 mutant phenotypes ${ }^{10,73}$. In addition, TF mutants generally have milder phenotypes than their upstream kinase and phosphatase mutants. For example, mutations in CRZ1, which encodes a downstream TF activated by Cnal phosphatase, result in much milder phenotypes than mutations in $C N A 1^{74}$. Thus, simple cophenotypic clustering of TFs, kinases, and phosphatases may lead to misinterpretations of the correlations between the signalling components. Functional and mechanistic relationships between signal components should be further investigated by RNA-seq, ChIP-seq, phosphoproteomics, and protein-protein interaction assays in future works.

Our systematic analysis unveiled both evolutionarily conserved and distinct sets of phosphatases in C. neoformans. Among 25 phosphatase genes that we were not able to disrupt, 14 genes (CDC25, SAC1, FCP1, IPC1, CET1, GLC7, TIM50, IDI1, IPP1, $R P P 1, H I S 2, M E T 22, P A H 1$, and GPI13) are known to be essential in S. cerevisiae, S. pombe, or C. albicans: (Supplementary Data 6). The following nine genes (CDC25, FCP1, IPC1, CET1, TIM50, $I D I 1, I P P 1, R P P 1$, and GPI13) are likely to be core essential fungal phosphatases because they are reported to be essential in $S$. cerevisiae and $S$. pombe. Out of 15 reported essential phosphatase genes in S. cerevisiae, we successfully deleted PHS1, CYR1 (Cac1 ortholog), CDC1, CDC14, SSU72, and DUT101 in C. neoformans. PHS1, CDC1, and DUT101 are also essential in S. pombe, suggesting that they are functionally divergent between ascomycete and basidiomycete fungi or have a synthetic lethal relationship with other functionally redundant phosphatases in C. neoformans. We have described similar findings in our previous analysis of kinase mutant collections in C. neoformans ${ }^{5}$. Therefore, it seems evident that some kinase/phosphatase-mediated signalling networks are functionally divergent among fungi.

Here, we unravelled 31 pathogenicity-related phosphatases that affect a variety of biological processes, including growth, virulence factor production, stress response, carbohydrate metabolism, cell signalling, protein sorting, and vesicular trafficking. Some of these findings are corroborated by previous research, including the role of PTP2, CAC1, CNA1, CCR4, and HAD1 in the virulence of $C$. neoformans ${ }^{10,11,13,15,16}$. Furthermore, in examining phosphatase mutants in a large deletion mutant analysis ${ }^{24}$, Liu et al. demonstrated that 4 of 14 mutants (cac1 $1, p t c 2 \Delta, s d p 102 \Delta$, and mre $11 \Delta)$ show reduced infectivity in the lungs ${ }^{24}$, consistent with our findings in $c a c 1 \Delta, s d p 102 \Delta$, and mre11 $\Delta$ mutants. Notably, Madhani's and our studies indicate that the $y v h 1 \Delta$ mutant is as infective as the wild-type strain as quantified by lung STM analysis, but, in our study, the $y v h 1 \Delta$ mutant exhibited attenuated virulence in the insect-killing model. Another deviation from prior research is the secreted acid phosphatase $A P H 1$, which was reported by Lev et al. to contribute to the virulence of $C$. neoformans ${ }^{18}$, but we found that deletion of $A P H 1$ did not significantly contribute to virulence in either the insect-killing assay or murine infection model. We attribute this discrepancy to the fact that Lev et al. incubated infected $G$. mellonella at $30^{\circ} \mathrm{C}$; because when we incubated insect hosts at $37^{\circ} \mathrm{C}$, the impact of thermotolerance at mammalian temperatures may have offset any advantages seen at lower temperatures ${ }^{18}$. For the remaining 23 phosphatases reported here, we are the first to describe their role in the pathogenicity of $C$. neoformans.

In this study, we found that a majority of the 31 pathogenicityrelated phosphatases were involved in regulation of three major cryptococcal virulence factors: thermotolerance, capsule, and melanin. Fourteen of them (Cna1, Gua1, Yvh1, Phs1, Nem1, Ppg1, Siw14, Inp5201, Ccr4, Dbr1, Ssu72, Mre11, Fbp26, and Tps2) promote thermotolerance at $37^{\circ} \mathrm{C}$. In addition, 13 phosphatases (Mre11, Ccr4, Vps29, Yvh1, Fbp26, Inp5201, Cac1, Ptp2, Dbr1, Ppg1, Nem1, Gua1, and Siw14) are involved in melanin production. Finally, capsule production was altered by deletion of six phosphatases: Cac1, Sdp102, Ptp2, Ynd1, Psr1, and Inp5201. Therefore, a total of 20 phosphatases are involved in regulation of any of the three major virulence factors, and 12 of them (Ccr4, Yvh1, Fbp26, Inp5201, Cac1, Ptp2, Dbr1, Ppg1, Nem1, Gua1, Mre11, and Siw14) are involved in modulating more than two virulence factors. The remaining 11 phosphatases (Ymr1, Oca1, Cdc1, Xpp1, Sdp101, Oca101, Gda1, Pph3, Had1, Sit4, and Inp5202) were unrelated to thermotolerance or capsule/melanin synthesis. Among these, Sdp101 and Inp5202 are paralogous to Sdp102 and Inp5201, respectively, but we failed to obtain corresponding double mutants, suggesting that Sdp101/Sdp102 and Inp5201/Inp5202 may have a synthetic lethal relationship and play redundant roles in $C$. neoformans. 
The pathobiological functions of Ymr1, Cdc1, Had1, and Pph3 remain unclear because mutants of these genes do not demonstrate clear phenotypic traits related to $C$. neoformans pathogenicity. However, the $y m r 1 \Delta, c d c 1 \Delta$, and $h a d 1 \Delta$ mutants showed increased susceptibility to cell membrane disruption, and this instability may have contributed to reduced infectivity or virulence relative to WT. The had $1 \Delta$ mutant also exhibited increased susceptibility to cell wall-destabilizing agents, which is in good agreement with recent reports showing that Hadl is a potential target of calcineurin, which plays a role in regulating cell wall integrity $^{74}$. Had1 was also shown to be required for virulence in a murine model of systemic cryptococcosis ${ }^{16}$. YMR1 encodes a phosphatidylinositol 3-phosphate (PI3P) phosphatase involved in vacuolar protein sorting pathways that functionally overlaps with other lipid phosphatases in S. cerevisiae. Therefore, deletion of YMR1 does not generate evident phenotypes in the yeast model ${ }^{75,76}$. Cdc1 is a putative mannose-ethanolamine phosphatase phosphodiesterase involved in both cell division cycle and GPI-anchor remodelling ${ }^{77,78}$. The role of Ymrl and Cdc1 in vacuolar protein sorting and GPI remodelling, respectively, may contribute to membrane stability in C. neoformans. In $S$. cerevisiae, $\mathrm{Pph} 3$ is a catalytic subunit of the protein phosphatase PP4 complex and governs recovery from the DNA damage checkpoint $^{79,80}$. Pph 3 likely plays a similar role in C. neoformans because the only in vitro phenotype observed in the pph3s mutant was increased susceptibility to DNA damage by methyl methane sulfonate (MMS). Given our previous demonstration that the DNA damage response pathway is critical for the virulence of C. neoformans ${ }^{81,82}$, we hypothesize the role of $\mathrm{Pph} 3$ in the DNA damage response likewise contributes to virulence.

We recently identified a group of $C$. neoformans TFs and kinases involved in brain infection processes, including $\mathrm{BBB}$ adhesion and crossing 39 . Here we found that the following pathogenicity-related phosphatases are also involved in BBB crossing of C. neoformans: Xpp1, Sit4, Ssu72, Siw14, and Gda1. In S. cerevisiae, Ppx1 (the orthologue of Xpp1) is a metal-dependent cytosolic expolyphosphatase and generates inorganic phosphate by cleaving polyphosphate that serves as a major phosphate reservoir and is required for long-term cell survival, gene regulation, cell motility, and stress responses ${ }^{83}$. The functions of Sit4 and Ssu72 are likely related to the target of rapamycin (TOR) pathway involved in nutrient-sensing. In S. cerevisiae, Sit4 is regulated by the nutrient-sensing TOR pathway ${ }^{84}$ and controls nitrogen catabolite repression genes through the GATA TF $\mathrm{Gln} 3^{85}$. Prior to transcriptional termination, Ssu72 dephosphorylates the C-terminal domain of the RNA polymerase II, which is degraded through the TOR-signalling pathway in response to rapamycin ${ }^{86}$. Accordingly, our sit4 $\Delta$ and ssu $72 \Delta$ mutants indeed exhibited highly increased susceptibility to rapamycin (Supplementary Fig. 13). Notably, we found that Sit4 and Ssu72 promote induction of the inositol transporter Itr $3 \mathrm{c}$ and metalloprotease Mpr1, respectively, required for BBB crossing of C. neoformans $s^{40,41}$. In S. cerevisiae, Siw14 hydrolyses the $\beta$ phosphatate from 5-diphosphoinositol pentakisphosphate $\left(\mathrm{IP}_{7}\right)$, and its deletion increases $\mathrm{IP}_{7}$ and decreases $\mathrm{IP}_{6}$ levels $^{87}$. Because deletion of the inositol pentakisphosphate kinase IPK1 decreases $\mathrm{IP}_{6}$ levels, abolishing the virulence of C. neoformans ${ }^{5,88}$, it is likely that Siw14 could promote BBB crossing and virulence of $C$. neoformans through modulation of the inositol signalling pathway. Finally, Gdal is a Golgi membrane-bound apyrase that modulates $O$-mannosylation in yeast ${ }^{34,35}$. We previously reported that the extended structure of $O$-glycans by mannose addition in the Golgi is required for cell integrity and full pathogenicity of $C$. neoformans ${ }^{38}$. Based on these observations, we conclude that phosphate utilization, TOR-mediated nutrient sensing, inositol- signalling, and $O$-mannosylation are critical for $\mathrm{BBB}$ crossing and brain infection.

The 31 pathogenicity-related phosphatases that were identified here and in previous studies could be potential antifungal drug targets, especially Sit4, Cna1, and Tps2, which are required for virulence of the major human fungal pathogens $C$. neoformans, $C$. albicans, and A. fumigatus. Sit4, Yvh1, Sdp101, Ptp2, Cac1, Tps2, Inp5201, and Ppg1 are required for virulence of both human and plant fungal pathogens, thus could be targets for the development of broad-spectrum antifungal drugs. Tps2 is a particularly promising antifungal drug target because the trehalose pathway is missing in humans ${ }^{89}$. Recently, the structure of the N-terminal domain of C. albicans Tps2 has been resolved ${ }^{90}$ and provides a structural basis for the design of Tps2-specific antifungal agents. Siw14, Had1, and Oca1/101 could also be good targets for cryptococcal treatment because there are no evident orthologues of these phosphatases in humans. Recently, the structure of the $S$. cerevisiae inositol phosphatase Siw14 has also been resolved ${ }^{91}$, whereas structural information for Had1 and Oca1/Oca101 is not yet available.

Several clinical strategies have targeted phosphatases directly by targeting the catalytic subunit of phosphatase complexes or indirectly by targeting the regulatory or scaffolding subunits of phosphatase complexes. Examples of the former strategy include LB-100 inhibition of the PP2A-C subunit ${ }^{92}$, FK506 inhibition of the calcineurin complex ${ }^{93-95}$, and inhibition of dual-specificity phosphatases by several small molecules ${ }^{96}$. An example of the latter strategy includes ceramide and its derivatives, which are bioactive sphingolipid molecules that activate PP2A by preventing SET inhibitor binding to PP2A ${ }^{97,98}$. To increase the specificity of a drug that targets a phosphatase, both the catalytic and regulatory subunits of the phosphatase complex should be considered, such as was considered with the drug FK506, which targets both the catalytic and regulatory subunits of the calcineurin complex via FKBP $12^{95}$. Based on BLASTP analysis using annotated information from the Saccharomyces Genome Database (SGD, http://yeastgenome.org), we found 26 putative phosphatase regulatory subunit orthologues in C. neoformans (Supplementary Data 10). However, further research is needed to characterize the functional and mechanistic relationships between the catalytic and regulatory subunits of phosphatases; this research could lead to the development of more antifungal drugs that target phosphatases.

\section{Methods}

Ethics statement. Animal care and all experiments were conducted in accordance with the ethical guidelines of the Institutional Animal Care and Use Committee (IACUC) of Yonsei University. The Yonsei University IACUC approved all vertebrate studies.

Construction of the $\boldsymbol{C}$. neoformans phosphatase and retromer mutants. We constructed phosphatase and retromer mutant strains in the C. neoformans serotype A H99S strain background through homologous recombination using genedisruption cassettes containing the nourseothricin-resistance marker (nourseothricin acetyl transferase; NAT) using NAT-split marker/double joint PCR (DJ-PCR) strategies ${ }^{99}$ (see Supplementary Data 4 for primers). We amplified $5^{\prime}$ and $3^{\prime}$-flanking regions of target genes by PCR with primer pairs L1/ L2 and R1/R2, respectively, from H99S genomic DNA. The signature-tagged NAT marker was amplified by PCR with primers M13Fe (M13 forward extended) and M13Re (M13 reverse extended) from a pNAT-STM plasmid containing the NAT gene with each unique, signature-tagged sequence. After amplification of the $5^{\prime}$ - and $3^{\prime}$-flanking regions and NAT marker in the first round of PCR to generate template DNA, the second round of PCR constructed $5^{\prime}$ - and $3^{\prime}$-regions of the NAT-split genedisruption cassette with primer pairs L1/NSL and R2/NSR, respectively. Biolistic transformation introduced the NAT gene-disruption cassettes by incubating the H99S strain in $50 \mathrm{ml}$ YPD medium for $16 \mathrm{~h}$ at $30^{\circ} \mathrm{C}$, followed by spin-down, resuspension in $5 \mathrm{ml}$ distilled water, application on YPD agar medium containing 1 $\mathrm{M}$ sorbitol, and further incubation for $3 \mathrm{~h}$ at $30^{\circ} \mathrm{C}$. Gene-disruption cassettes were then combined with $600 \mu \mathrm{g}$ of $0.6-\mu \mathrm{m}$ gold microcarrier beads (Bio-Rad Laboratories, Hercules, CA, USA) and introduced into cells using a particle delivery 
system (PDS-100, Bio-Rad). After $4 \mathrm{~h}$ incubation at $30^{\circ} \mathrm{C}$ for recovery of cell membrane integrity, cells were scraped and spread on YPD agar medium containing nourseothricin $(100 \mu \mathrm{g} / \mathrm{ml})$. Diagnostic PCR identified NAT-positive transformants. Southern blot analysis confirmed the genotype of each screened transformant, and we constructed at least two independent mutant strains for each phosphatase gene.

nCounter in vivo gene expression profiling analysis. Using previously reported RNA samples ${ }^{39}$, we performed an nCounter gene expression analysis (NanoString) to quantify in vivo expression levels of the 139 phosphatases. Total RNA samples were obtained from 6 -week-old female A/J mice infected with $5 \times 10^{5}$ cells through nasal inhalation. Each group of three mice was sacrificed 3, 7, 14, or $21 \mathrm{dpi}$, and the lungs, brain, spleen, and kidneys were recovered and lyophilized. Dried organs were homogenized, and total RNA was extracted with a commercial RNA extraction kit (easy-BLUE, Intron Biotechnology). Samples containing $10 \mathrm{ng}$ of total RNA isolated from C. neoformans grown under in vitro basal conditions $\left(30^{\circ} \mathrm{C}\right.$; YPD medium) or $10 \mu \mathrm{g}$ of total RNA isolated from C. neoformans-infected mouse tissues were reacted with the custom-designed probe code set according to the manufacturer's standard protocol of the nCounter multiplex platform (NanoString, Seattle, WA, USA ${ }^{22,23}$. Scanning was performed by digital analyser through high resolution (600 fields) option and normalized by nSolver software (provided by NanoString). A total of eight housekeeping genes (mitochondrial protein, CNAG_00279; microtubule-binding protein, CNAG_00816; aldose reductase, CNAG_02722; cofilin, CNAG_02991; actin, CNAG_00483; tubulin $\beta$ chain, CNAG_01840; tubulin $\alpha$-1A chain, CNAG_03787; histone H3, CNAG_04828) were used for expression normalization ${ }^{39,100}$. Normalized data were transformed to $\log _{2}$ scores to express fold-change and subjected to clustering using one minus Pearson correlation with average linkage by Morpheus (Broad Institute, Cambridge, MA, USA, http://software.broadinstitute.org/morpheus).

Growth and chemical susceptibility test. To analyse the susceptibility of each phosphatase mutant to in vitro stress conditions, C. neoformans was grown for $16 \mathrm{~h}$ at $30^{\circ} \mathrm{C}$, serially diluted 10 -fold $\left(1-10^{4}\right)$, and spotted on YPD agar media containing the following chemical agents to induce environmental stress as described previously 4,5 : osmotic stress (sorbitol) and cation/salt stresses $(\mathrm{NaCl}$ and $\mathrm{KCl}$ ) under either glucose-rich (YPD) or glucose-starved (yeast extract-peptone; YP) conditions; oxidative stress [hydrogen peroxide $\left(\mathrm{H}_{2} \mathrm{O}_{2}\right)$, tert-butyl hydroperoxide (an organic peroxide), menadione (a superoxide anion generator), diamide (a thiolspecific oxidant)]; toxic heavy metal stress [cadmium sulfate $\left(\mathrm{CdSO}_{4}\right)$ ]; genotoxic stress (methyl methanesulphonate and hydroxyurea); membrane destabilizing stress [sodium dodecyl sulfate (SDS)]; cell wall destabilizing stress (calcofluor white and Congo red); ER stress [tunicamycin and dithiothreitol (DTT)]; and antifungal drug susceptibility (fludioxonil, fluconazole, amphotericin B, and flucytosine). Cells were incubated at $30^{\circ} \mathrm{C}$ for $1-5$ days and photographed daily. To examine the growth of $\mathrm{C}$. neoformans strains at different temperatures, we spotted serially diluted cells on YPD agar medium, incubated them at 25,37 , and $39^{\circ} \mathrm{C}$, and photographed the cultures daily. To quantitatively examine the growth rate of phosphatase mutants, the WT strain (H99S) and phosphatase mutants were incubated at $30^{\circ} \mathrm{C}$ overnight and sub-cultured into fresh liquid YPD medium [optical density at $600 \mathrm{~nm}\left(\mathrm{OD}_{600}\right)=0.2$ ]. Cells were than incubated at 30 or $37^{\circ} \mathrm{C}$ in a multi-channel bioreactor (Biosan Laboratories, Inc., Warren, MI, USA), and $\mathrm{OD}_{600 \mathrm{~nm}}$ was automatically measured for $40-90 \mathrm{~h}$.

Mating assay. To examine unilateral mating efficiency, each serotype A MATa phosphatase mutant constructed in the H99S strain and MATa KN99a strain were cultured in YPD medium for $16 \mathrm{~h}$ at $30^{\circ} \mathrm{C}$, washed twice with phosphate-buffered saline (PBS), mixed at equal concentrations $\left(10^{7} \mathrm{cells} / \mathrm{ml}\right)$, spotted on V8 mating media ( $\mathrm{pH}$ 5), and incubated at $25^{\circ} \mathrm{C}$ in the dark for 7-14 days. Filamentous growth was observed and photographed weekly.

In vitro virulence factor production assay. To test capsule production efficiency, each mutant was cultured at $30^{\circ} \mathrm{C}$, spotted onto Dulbecco's modified Eagle's (DME) agar medium, and incubated at $37^{\circ} \mathrm{C}$ for 2 days, after which cells were scraped, washed with distilled water, fixed with $10 \%$ formalin, and washed again with distilled water. Fixed cells were adjusted to a concentration of $3 \times 10^{8} \mathrm{cells} / \mathrm{ml}$, and $50 \mu \mathrm{l}$ of the cell suspension was injected into microhaematocrit capillary tubes (Kimble Chase, Rockwood, TN, USA). Capillary tubes were placed vertically for 10 days to pack cells by gravity. Packed cell volume ratio (packed cell phase/total phase) was measured, and the relative packed cell volume of each mutant was calculated by normalizing each ratio with packed cell volume ratio of wild-type H99S strain. Statistical differences in relative packed cell volume ratios were determined by one-way analysis of variance (ANOVA) with Bonferroni's multiple comparison test in Prism 8 (GraphPad, San Diego, USA). To measure capsule production efficiency in other media, $5 \mu \mathrm{l}$ of each culture was spotted on Littman's agar medium ${ }^{101}$ and FBS agar medium (10\% of foetal bovine serum and $90 \%$ of PBS), incubated at $37^{\circ} \mathrm{C}$ for 2 days, scraped, and resuspended with distilled water. Resuspended cells were stained by India ink (BactiDrop; Remel, San Diego, CA, USA) and observed by differential interference contrast (DIC) microscopy (BX51, Olympus, Tokyo, Japan). Capsule thickness was measured by subtracting the cell diameter from capsule diameter (total diameter-cell body diameter). For quantitative measurement of capsule thickness, 50 cells were measured for the H99S strain and each phosphatase mutant. To examine melanin production efficiency, each phosphatase mutant was cultured for $16 \mathrm{~h}$ in YPD medium at $30^{\circ} \mathrm{C}$, washed with PBS, and spotted $(3 \mu \mathrm{l})$ on Niger seed, dopamine, or epinephrine media $(1 \mathrm{~g}$ L-asparagine, $3 \mathrm{~g} \mathrm{KH}_{2} \mathrm{PO}_{4}, 250 \mathrm{mg} \mathrm{MgSO}{ }_{4}, 1 \mathrm{mg}$ thiamine, $5 \mu \mathrm{g}$ biotin, and $100 \mathrm{mg}$ L-DOPA or epinephrine hydrochloride per litter) containing $0.1 \%$ or $0.2 \%$ glucose Spotted cells were incubated at $37^{\circ} \mathrm{C}$ and photographed after 1-3 days. For the phosphatase mutants growth-defective at $37^{\circ} \mathrm{C}$, melanin and capsule production efficiency were examined at $30^{\circ} \mathrm{C}$. To examine urease production, each phosphatase mutant was cultured at $30^{\circ} \mathrm{C}$ for $16 \mathrm{~h}$, washed with PBS, and inoculated $\left(10^{6}\right.$ cells) onto liquid Christensen's media in a $10-\mathrm{ml}$ medical tube (SPL Life Sciences, Gyeonggi, Korea), then incubated at $30^{\circ} \mathrm{C}$ in a shaking incubator for $1-3$ days and photographed daily.

Expression analysis. To measure the expression level of known melaninregulating genes (LAC1, HOB1, MBS1, and BZP4), the H99S strain and phosphatase mutants (mre 11 $\Delta, c c r 4 \Delta, v p s 29 \Delta, y v h 1 \Delta$, fbp26 ptc $2 \Delta, d b r 1 \Delta, p p g 1 \Delta$, nem $1 \Delta$, and gua $1 \Delta$ ) were incubated in liquid YPD medium for $16 \mathrm{~h}$ at $30^{\circ} \mathrm{C}$ and sub-cultured into fresh liquid YPD medium $\left(\mathrm{OD}_{600}=0.2\right)$. When the cells reached the early logarithmic phase $\left(\mathrm{OD}_{600}=0.6-0.8\right)$, half of the cell culture was sampled to prepare a basal sample. The remaining cell culture was washed three times with PBS and incubated in nutrient-starvation conditions (YNB medium with ammonium sulfate without glucose) for $2 \mathrm{~h}$, then immediately pelleted with liquid nitrogen and lyophilized. To measure the expression level of $\mathrm{BBB}$ crossing-related genes (ITR1A, ITR3C, MPR1, FZC31, GAT201, and PDR802), the

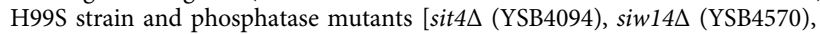
ssu72 $\Delta$ (YSB4242), gda1 $\Delta$ (YSB4750), and $x p p 1 \Delta$ (YSB5941)] were incubated in YPD broth at $30^{\circ} \mathrm{C}$ for $16 \mathrm{~h}$, sub-cultured into $50 \mathrm{ml}$ of fresh YPD broth, and further incubated until $\mathrm{OD}_{600}$ reached 0.8 . The culture was then separated into two 25 - $\mathrm{ml}$ tubes, centrifuged, and washed three times with sterile distilled water. One tube was kept in liquid nitrogen to monitor basal expression levels, and the other tube was resuspended with an equal volume of RPMI1640 medium containing 10\% FBS. After $3 \mathrm{~h}$ incubation at $37^{\circ} \mathrm{C}$ in a $\mathrm{CO}_{2}$ incubator with $120 \mathrm{rpm}$ horizontal shaking, cells were centrifuged and lyophilized overnight. Total RNA was extracted from each sample using a commercial RNA extraction kit (easy-BLUE, iNtRON Biotechnology, Gyeonggi, Korea) and cDNA was synthesized using RTase (Thermo Scientific, Waltham, MA, USA). Quantitative reverse transcription-PCR (qRTPCR) was performed with target gene-specific primer pairs listed in Supplementary Data 4.

HPLC analysis of $\mathbf{0}$-linked glycans from cwMPs. Analysis of $O$-linked glycans from cwMPs was conducted as described previously 38,102 . The $O$-linked oligosaccharides were released from the purified cwMPs by modified hydrazinolysis. Dried cwMPs $(50 \mu \mathrm{g})$ were resuspended in hydrazine monohydrate and incubated at $60^{\circ} \mathrm{C}$ for $4 \mathrm{~h}$. After cooling and desiccation of the reactant, the pellets were dissolved in $\mathrm{NaHCO}_{3}$, mixed with $\left(\mathrm{CH}_{3} \mathrm{CO}\right)_{2} \mathrm{O}$, and incubated on ice for $30 \mathrm{~min}$ $\mathrm{O}$-glycans were purified by Dowex 50WX8-400 resins (Sigma-Aldrich, St. Louis, MO, USA) and labelled with 2-AA. The purified $O$-glycan was analysed using HPLC on a TSKgel Amide- 80 column $(0.46 \times 25 \mathrm{~cm}$, Tosoh Corp., Tokyo, Japan with $90 \%$ solvent A ( $2 \%$ acetic acid and $1 \%$ tetrahydrofuran in acetonitrile) and $10 \%$ solvent B (5\% acetic acid, $3 \%$ triethylamine, and $1 \%$ tetrahydrofuran in water) After sample injection, the proportion of solvent B increased to $90 \%$ over $60 \mathrm{~min}$ at a flow rate of $1.0 \mathrm{ml} / \mathrm{min}$ and then $\mathrm{O}$-glycans were detected with a fluorescence detector (2475, Waters Corp., Milford, MA, USA) at excitation and emission wavelengths of 360 and $425 \mathrm{~nm}$, respectively. Data were analysed by using chromatography software (Empower 2, Waters).

Insect-based in vivo virulence assay. At least $15 \mathrm{G}$. mellonella caterpillars (Vanderhorst Wholesale, Inc., Saint Marys, OH, USA) in the final larval instar with a body weight of 200-300 mg, arriving within 7 days from the day of shipment, were used for in vivo insect virulence assays. Each phosphatase mutant and wildtype $\mathrm{H} 99 \mathrm{~S}$ strain was incubated at $30^{\circ} \mathrm{C}$ overnight, pelleted, washed three times with PBS, and resuspended in PBS at a concentration of $10^{6}$ cells $/ \mathrm{ml}$. Four thousand C. neoformans cells per larvae were injected into the second-to-last prolegs with a $100-\mu \mathrm{l}$ syringe equipped with a $10-\mu \mathrm{l}$ needle and repeating dispenser (PB600-1, Hamilton Company, Reno, NV, USA). Negative control G. mellonella received PBS only. Infected larvae were placed in Petri dishes in a humidified container, incubated at $37^{\circ} \mathrm{C}$, and monitored daily. Larvae were considered dead when they turned black and showed no movement upon touching. Larvae that pupated during the experiment were censored for statistical analysis. Survival curves were illustrated using Prism 8 (GraphPad, San Diego, CA, USA) and analysed with a log-rank (Mantel-Cox) test. We examined two independent strains for each phosphatase mutant.

STM-based murine infectivity assay. A set of phosphatase mutants with the 41 unique signature-tagged $N A T$ selection markers was cultured for $16 \mathrm{~h}$ at $30^{\circ} \mathrm{C}$. The

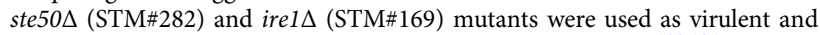
avirulent control strains, respectively, as described previously 103,104 . Mutants and 
control strains were pelleted, washed three times with PBS, resuspended in PBS, and then pooled at equal numbers $\left(5 \times 10^{5}\right.$ cells). Seven-week-old female A/J mice (Jackson Laboratory, Bar Harbor, ME, USA) anaesthetized with an intraperitoneal injection of Avertin (2,2,2-tribromoethanol, T48402, Sigma-Aldrich, St. Louis, MO, USA) were infected with $5 \times 10^{5}$ cells of pooled mutants (in $50 \mu \mathrm{l}$ PBS) through intranasal inhalation. To prepare the input phosphatase genomic DNA library, $200 \mu \mathrm{l}$ of pooled strains were spread on YPD media containing $100 \mu \mathrm{g} / \mathrm{ml}$ chloramphenicol, incubated at $30^{\circ} \mathrm{C}$ for 3 days, and collected by scraping. The infected mice were sacrificed with an overdose of Avertin $14 \mathrm{dpi}$. Lungs and brains of infected mice were recovered and homogenized with $5 \mathrm{ml}$ of PBS. Homogenized tissues were then spread on YPD medium containing $100 \mu \mathrm{g} / \mathrm{ml}$ chloramphenicol, incubated at $30^{\circ} \mathrm{C}$ for 3 days, and collected by scraping. Genomic DNA was extracted from collected input and output cells using the cetyl trimethylammonium bromide $(\mathrm{CTAB})$ method ${ }^{103}$. Quantitative PCR was performed with tag-specific primers (Supplementary Data 4) using a qRT-PCR system (CFX96, Bio-Rad, Hercules, CA, USA). The STM score was calculated using the $2^{-\Delta \Delta C T}$ method ${ }^{105}$ to determine STM score to calculate the relative changes in genomic DNA amounts. The mean fold-changes in input verses output samples were calculated as a log score $\left(\log _{2} 2^{-(C t, T a r g e t-C t, A c t i n)}\right)$ output-(Ct,Target-Ct,Actin)input)

In vitro BBB-crossing and BBB-adhesion assays. Human brain microvascular endothelial cells (hCMEC/D3 cell line, Merck \& Co., Kenilworth, NJ, USA) were cultured as follows based on the reported methods $\mathrm{s}^{39,106,107}$. Briefly, $5 \times 10^{4}$ hCMEC/D3 cells in EGM-2 medium (Lonza Group, Basel, Switzerland) were seeded on collagen (Corning, Inc., Corning, NY, USA)-coated $8-\mu \mathrm{m}$ porous membranes (BD Biosciences, Franklin Lakes, NJ, USA) for the BBB-crossing assay or 12-well plates (BD Biosciences) for the BBB-adhesion assay. The day after seeding, medium was refreshed with EGM-2 medium supplemented with $2.5 \%$ human serum and further incubated for 4 days. A day before C. neoformans inoculation, the medium was replaced with $0.5 \times$ diluted EGM- 2 medium, and the cells were maintained at $37{ }^{\circ} \mathrm{C}$ and $5 \% \mathrm{CO}_{2}$. The integrity of tight junctions between cells was confirmed by verifying that the trans-endothelial electrical resistance (TEER) measured $\sim 200 \Omega / \mathrm{cm}^{2}$, as assed by an epithelial volt/ohm meter $\left(\right.$ EVOM $^{2}$ device, World Precision Instruments, Sarasota, FL, USA).

For the BBB-crossing assay, $5 \times 10^{5}$ cells of $C$. neoformans WT (H99), mpr1 $1 \Delta$ mutant, and phosphatase deletion mutants were added to $500 \mu \mathrm{l}$ of PBS and inoculated onto the porous membranes. After $24 \mathrm{~h}$ incubation at $37^{\circ} \mathrm{C}$ in $5 \% \mathrm{CO}_{2}$, the number of yeast cells passing through the porous membrane was measured by counting CFU. Tight junction integrity was measured by TEER as described above before and after inoculation of yeast cells. The BBB migration ratio was calculated by dividing the output CFU of each tested strain by that of WT. For the BBBadhesion assay, $5 \times 10^{5}$ yeast cells in $100 \mu$ l of PBS were inoculated onto a monolayer of hCMEC/D3 cells grown in a 12-well plate and incubated for $24 \mathrm{~h}$ at $37^{\circ} \mathrm{C}$ in $5 \% \mathrm{CO}_{2}$. Following incubation, cultures were washed three times with PBS, incubated with sterile distilled water for $30 \mathrm{~min}$ at $37^{\circ} \mathrm{C}$ to burst the host cells, and collected for CFU quantification. The BBB-adhesion ratio was calculated by dividing the adhered CFU of each test strain by that of WT C. neoformans.

\section{C. neoformans phosphatase web-accessible database. We developed the} Cryptococcus neoformans Phosphatase Phenome Database (http://phosphatase. cryptococcus.org) and Cryptococcus neoformans Phenome Gateway Database (http://www.cryptococcus.org/) to facilitate public access to the phenomic and genomic data for the C. neoformans phosphatase mutant library. The database was built under the same software development environment as described in our two previous publications ${ }^{4,5}$. Genome sequences and annotations of the H99S strain were obtained from the standardized genome data warehouse in the Comparative Fungal Genomics Platform Database (CFGP 2.0; http://cfgp.riceblast.snu.ac.kr) $)^{108}$. Except reported phosphatases, those first functionally characterized by this study were named based on the published gene nomenclature rules for C. neoformans ${ }^{109}$ (Fig. 1a).

Statistical analysis. Statistical analyses were performed with GraphPad Prism version 8. For the capsule production assay and expression analysis, ANOVA with Bonferroni's multiple comparison test was used. For the insect-killing assay, the log-rank (Mantel-Cox) test was used for statistical analysis. For the murine STM analysis, the statistical significance between ste50 $\Delta$ (positive control) and mutants was calculated by one-way ANOVA with Bonferroni's multiple comparisons test.

Reporting summary. Further information on research design is available in the Nature Research Reporting Summary linked to this article.

\section{Data availability}

The phosphatase domain and sequence data in Fig. 1 were retrieved from FungiDB (https://fungidb.org/fungidb/) and InterPro (https://www.ebi.ac.uk/interpro/). All data to classify the phosphatases in Fig. 1a and b are available in Supplementary Data 1. Detailed information about phosphatases in S. cerevisiae, S. pombe, C. albicans and U. maydis is listed in Supplementary Data 3. Information about gene IDs and names, strain numbers, and genotypes with signature tag numbers for phosphatase mutant strains used in this study is available in Supplementary Data 5. We provide the whole phosphatase mutant collection via Fungal Genetics Stock Center (FGSC, http://www.fgsc.net/) in USA, Korean Culture Center of Microorganisms (KCCM, http://www.kccm.or.kr/) and Korean Collection for Type Cultures (KCTC, https://kctc.kribb.re.kr/) in South Korea. The whole phenome data for phosphatase mutants are available in the Cryptococcus neoformans Phosphatase Phenome Database (http://phosphatase.cryptococcus.org). Integrated phenome data of transcription factor, kinase, and phosphatase mutants in C. neoformans in this and previous studies ${ }^{4,5}$ are available in the Cryptococcus neoformans Phenome Gateway Database (http://www.cryptococcus.org/), in which individual gene/protein information is linked to that of FungiDB. The whole NanoString-nCounter ${ }^{\oplus}$ analysis data for in vivo phosphatase gene expression and probe information are available in Supplementary Data 8 . The source data underlying Supplementary Figs. 7b, 11b, d, f and $\mathrm{h}$ are provided as a Source Data file. Source data are provided with this paper.

Received: 17 February 2020; Accepted: 30 July 2020; Published online: 24 August 2020

\section{References}

1. Bononi, A. et al. Protein kinases and phosphatases in the control of cell fate. Enzym. Res. 2011, 329098 (2011).

2. Bahn, Y. S. \& Jung, K. W. Stress signaling pathways for the pathogenicity of Cryptococcus. Eukaryot. Cell 12, 1564-1577 (2013).

3. Rajasingham, R. et al. Global burden of disease of HIV-associated cryptococcal meningitis: an updated analysis. Lancet Infect. Dis. 17, 873-881 (2017).

4. Jung, K. W. et al. Systematic functional profiling of transcription factor networks in Cryptococcus neoformans. Nat. Commun. 6, 6757 (2015).

5. Lee, K. T. et al. Systematic functional analysis of kinases in the fungal pathogen Cryptococcus neoformans. Nat. Commun. 7, 12766 (2016).

6. Blaskovich, M. A. Drug discovery and protein tyrosine phosphatases. Curr. Med. Chem. 16, 2095-2176 (2009).

7. Winkelstroter, L. K. et al. Systematic global analysis of genes encoding protein phosphatases in Aspergillus fumigatus. G3: Genes Genomes Genet. 5 , 1525-1539 (2015).

8. Yun, Y. et al. Functional analysis of the Fusarium graminearum phosphatome. New Phytol. 207, 119-134 (2015).

9. Park, H. S., Lee, S. C., Cardenas, M. E. \& Heitman, J. Calcium-calmodulincalcineurin signaling: a globally conserved virulence cascade in eukaryotic microbial pathogens. Cell Host Microbe 26, 453-462 (2019).

10. Lee, K. T. et al. Distinct and redundant roles of protein tyrosine phosphatases Ptp1 and Ptp2 in governing the differentiation and pathogenicity of Cryptococcus neoformans. Eukaryot. Cell 13, 796-812 (2014).

11. Alspaugh, J. A. et al. Adenylyl cyclase functions downstream of the Galpha protein Gpal and controls mating and pathogenicity of Cryptococcus neoformans. Eukaryot. Cell 1, 75-84 (2002).

12. Lee, K. T., Kwon, H., Lee, D. \& Bahn, Y. S. A nudix hydrolase protein, Ysa1, regulates oxidative stress response and antifungal drug susceptibility in Cryptococcus neoformans. Mycobiology 42, 52-58 (2014).

13. Odom, A. et al. Calcineurin is required for virulence of Cryptococcus neoformans. EMBO J. 16, 2576-2589 (1997).

14. Petzold, E. W. et al. Characterization and regulation of the trehalose synthesis pathway and its importance in the pathogenicity of Cryptococcus neoformans. Infect. Immun. 74, 5877-5887 (2006).

15. Panepinto, J. C. et al. Binding of serum mannan binding lectin to a cell integrity-defective Cryptococcus neoformans ccr $4 \Delta$ mutant. Infect. Immun. 75, 4769-4779 (2007)

16. Jung, W. H. et al. Had1 is required for cell wall integrity and fungal virulence in Cryptococcus neoformans. G3: Genes Genomes Genet. 8, 643-652 (2018).

17. Kretschmer, M. et al. Defects in phosphate acquisition and storage influence virulence of Cryptococcus neoformans. Infect. Immun. 82, 2697-2712 (2014).

18. Lev, S. et al. Identification of Aph1, a phosphate-regulated, secreted, and vacuolar acid phosphatase in Cryptococcus neoformans. mBio 5, e01649-01614 (2014).

19. Lev, S. et al. Fungal inositol pyrophosphate $\mathrm{IP}_{7}$ is crucial for metabolic adaptation to the host environment and pathogenicity. mBio 6, e00531-00515 (2015).

20. Shea, J. M., Kechichian, T. B., Luberto, C. \& Del Poeta, M. The cryptococcal enzyme inositol phosphosphingolipid-phospholipase $\mathrm{C}$ confers resistance to the antifungal effects of macrophages and promotes fungal dissemination to the central nervous system. Infect. Immun. 74, 5977-5988 (2006).

21. Gerik, K. J. et al. Cell wall integrity is dependent on the PKC1 signal transduction pathway in Cryptococcus neoformans. Mol. Microbiol. 58, 393-408 (2005).

22. Fanning, S. et al. Divergent targets of Candida albicans biofilm regulator Bcr 1 in vitro and in vivo. Eukaryot. Cell 11, 896-904 (2012). 
23. Xu, W., Solis, N. V., Filler, S. G. \& Mitchell, A. P. Pathogen gene expression profiling during infection using a NanoString nCounter platform. Methods Mol. Biol. 1361, 57-65 (2016).

24. Liu, O. W. et al. Systematic genetic analysis of virulence in the human fungal pathogen Cryptococcus neoformans. Cell 135, 174-188 (2008).

25. Zaragoza, O., de Virgilio, C., Ponton, J. \& Gancedo, C. Disruption in Candida albicans of the TPS2 gene encoding trehalose-6-phosphate phosphatase affects cell integrity and decreases infectivity. Microbiology 148, 1281-1290 (2002).

26. Desai, J. V. et al. Coordination of Candida albicans invasion and infection functions by phosphoglycerol phosphatase Rhr2. Pathogens 4, 573-589 (2015).

27. Hanaoka, N. et al. Identification of the putative protein phosphatase gene PTC1 as a virulence-related gene using a silkworm model of Candida albicans infection. Eukaryot. Cell 7, 1640-1648 (2008).

28. Wang, Y., Aisen, P. \& Casadevall, A. Cryptococcus neoformans melanin and virulence: mechanism of action. Infect. Immun. 63, 3131-3136 (1995).

29. Kozel, T. R. \& Gotschlich, E. C. The capsule of Cryptococcus neoformans passively inhibits phagocytosis of the yeast by macrophages. J. Immunol. 129, 1675-1680 (1982).

30. Lee, D. et al. Unraveling melanin biosynthesis and signaling networks in Cryptococcus neoformans. mBio 10, e02267-19 (2019).

31. Seaman, M. N., McCaffery, J. M. \& Emr, S. D. A membrane coat complex essential for endosome-to-Golgi retrograde transport in yeast. J. Cell Biol. 142, 665-681 (1998).

32. Bonifacino, J. S. \& Hurley, J. H. Retromer. Curr. Opin. Cell Biol. 20, 427-436 (2008).

33. Seaman, M. N. Recycle your receptors with retromer. Trends Cell Biol. 15, 68-75 (2005).

34. Abeijon, C. et al. Guanosine diphosphatase is required for protein and sphingolipid glycosylation in the Golgi lumen of Saccharomyces cerevisiae. J. Cell Biol. 122, 307-323 (1993).

35. Gao, X. D., Kaigorodov, V. \& Jigami, Y. YND1, a homologue of GDA1, encodes membrane-bound apyrase required for Golgi $N$ - and $O$-glycosylation in Saccharomyces cerevisiae. J. Biol. Chem. 274, 21450-21456 (1999).

36. Yanagisawa, K., Resnick, D., Abeijon, C., Robbins, P. W. \& Hirschberg, C. B. A guanosine diphosphatase enriched in Golgi vesicles of Saccharomyces cerevisiae. Purification and characterization. J. Biol. Chem. 265, 19351-19355 (1990).

37. Herrero, A. B., Uccelletti, D., Hirschberg, C. B., Dominguez, A. \& Abeijon, C. The Golgi GDPase of the fungal pathogen Candida albicans affects morphogenesis, glycosylation, and cell wall properties. Eukaryot. Cell 1, 420-431 (2002).

38. Lee, D. J., Bahn, Y. S., Kim, H. J., Chung, S. Y. \& Kang, H. A. Unraveling the novel structure and biosynthetic pathway of $O$-linked glycans in the Golgi apparatus of the human pathogenic yeast Cryptococcus neoformans. J. Biol. Chem. 290, 1861-1873 (2015).

39. Lee, K. T. et al. Fungal kinases and transcription factors regulating brain infection in Cryptococcus neoformans. Nat. Commun. 11, 1521 (2020).

40. $\mathrm{Vu}, \mathrm{K}$. et al. Invasion of the central nervous system by Cryptococcus neoformans requires a secreted fungal metalloprotease. $m$ Bio $\mathbf{5}$, e01101-e01114 (2014).

41. Liu, T. B. et al. Brain inositol is a novel stimulator for promoting Cryptococcus penetration of the blood-brain barrier. PLoS Pathog. 9, e1003247 (2013).

42. Feng, J. et al. The N-terminal pY33XL motif of CaPsy2 is critical for the function of protein phosphatase 4 in CaRad53 deactivation, DNA damageinduced filamentation and virulence in Candida albicans. Int. J. Med. Microbiol. 307, 471-480 (2017).

43. Bader, T., Bodendorfer, B., Schroppel, K. \& Morschhauser, J. Calcineurin is essential for virulence in Candida albicans. Infect. Immun. 71, 5344-5354 (2003).

44. Lee, C. M., Nantel, A., Jiang, L., Whiteway, M. \& Shen, S. H. The serine/ threonine protein phosphatase SIT4 modulates yeast-to-hypha morphogenesis and virulence in Candida albicans. Mol. Microbiol. 51, 691-709 (2004).

45. Hanaoka, N. et al. A putative dual-specific protein phosphatase encoded by YVH1 controls growth, filamentation and virulence in Candida albicans. Microbiology 151, 2223-2232 (2005).

46. Csank, C. et al. Derepressed hyphal growth and reduced virulence in a VH1 family-related protein phosphatase mutant of the human pathogen Candida albicans. Mol. Biol. Cell 8, 2539-2551 (1997).

47. Noble, S. M., French, S., Kohn, L. A., Chen, V. \& Johnson, A. D. Systematic screens of a Candida albicans homozygous deletion library decouple morphogenetic switching and pathogenicity. Nat. Genet. 42, 590-598 (2010).

48. Rocha, C. R. et al. Signaling through adenylyl cyclase is essential for hyphal growth and virulence in the pathogenic fungus Candida albicans. Mol. Biol. Cell 12, 3631-3643 (2001).

49. Dagley, M. J. et al. Cell wall integrity is linked to mitochondria and phospholipid homeostasis in Candida albicans through the activity of the post-transcriptional regulator Ccr4-Pop2. Mol. Microbiol. 79, 968-989 (2011).
50. Badrane, $H$. et al. The Candida albicans phosphatase Inp51p interacts with the EH domain protein Irs4p, regulates phosphatidylinositol-4,5-bisphosphate levels and influences hyphal formation, the cell integrity pathway and virulence. Microbiology 154, 3296-3308 (2008).

51. Albataineh, M. T., Lazzell, A., Lopez-Ribot, J. L. \& Kadosh, D. Ppg1, a PP2Atype protein phosphatase, controls filament extension and virulence in Candida albicans. Eukaryot. Cell 13, 1538-1547 (2014).

52. Jiang, L. et al. Functional characterization and virulence study of $A D E 8$ and GUA1 genes involved in the de novo purine biosynthesis in Candida albicans. FEMS Yeast Res. 10, 199-208 (2010).

53. Steinbach, W. J. et al. Calcineurin controls growth, morphology, and pathogenicity in Aspergillus fumigatus. Eukaryot. Cell 5, 1091-1103 (2006).

54. Bom, V. L. et al. The Aspergillus fumigatus sitA phosphatase homologue is important for adhesion, cell wall integrity, biofilm formation, and virulence. Eukaryot. Cell 14, 728-744 (2015).

55. Puttikamonkul, S. et al. Trehalose 6-phosphate phosphatase is required for cell wall integrity and fungal virulence but not trehalose biosynthesis in the human fungal pathogen Aspergillus fumigatus. Mol. Microbiol. 77, 891-911 (2010)

56. Bormann, J., Boenisch, M. J., Bruckner, E., Firat, D. \& Schafer, W. The adenylyl cyclase plays a regulatory role in the morphogenetic switch from vegetative to pathogenic lifestyle of Fusarium graminearum on wheat. PLoS ONE 9, e91135 (2014).

57. Zheng, W. et al. Retrograde trafficking from the endosome to the trans-Golgi network mediated by the retromer is required for fungal development and pathogenicity in Fusarium graminearum. New Phytol. 210, 1327-1343 (2016).

58. Choi, J. H., Kim, Y. \& Lee, Y. H. Functional analysis of MCNA, a gene encoding a catalytic subunit of calcineurin, in the rice blast fungus Magnaporthe oryzae. J. Microbiol. Biotechnol. 19, 11-16 (2009).

59. Liu, X. et al. MoYvh1 subverts rice defense through functions of ribosomal protein MoMrt4 in Magnaporthe oryzae. PLoS Pathog. 14, e1007016 (2018).

60. Wang, R. J., Peng, J., Li, Q. X. \& Peng, Y. L. Phosphorylation-mediated regulatory networks in mycelia of Pyricularia oryzae revealed by phosphoproteomic analyses. Mol. Cell. Proteom. 16, 1669-1682 (2017)

61. Choi, W. \& Dean, R. A. The adenylate cyclase gene MAC1 of Magnaporthe grisea controls appressorium formation and other aspects of growth and development. Plant Cell 9, 1973-1983 (1997).

62. Long, B. et al. NTPDase specific inhibitors suppress rice infection by Magnaporthe oryzae. J. Phytopathol. 163, 227-232 (2015).

63. Wang, Y., Jiao, T. L., Liu, X. H., Lin, F. C. \& Wu, W. R. Functional characterization of a NEM1-like gene in Magnaporthe oryzae. Agr. Sci. China 10, 1385-1390 (2011).

64. Du, Y. et al. A serine/threonine-protein phosphatase PP2A catalytic subunit is essential for asexual development and plant infection in Magnaporthe oryzae. Curr. Genet. 59, 33-41 (2013).

65. Zheng, W. et al. Retromer is essential for autophagy-dependent plant infection by the rice blast fungus. PLoS Genet. 11, e1005704 (2015).

66. Di Como, C. J. \& Arndt, K. T. Nutrients, via the Tor proteins, stimulate the association of Tap42 with type 2A phosphatases. Genes Dev. 10, 1904-1916 (1996).

67. Kammer, G. M. The adenylate cyclase-cAMP-protein kinase A pathway and regulation of the immune response. Immunol. Today 9, 222-229 (1988).

68. Beeser, A. E. \& Cooper, T. G. The dual-specificity protein phosphatase Yvh1p regulates sporulation, growth, and glycogen accumulation independently of catalytic activity in Saccharomyces cerevisiae via the cyclic AMP-dependent protein kinase cascade. J. Bacteriol. 182, 3517-3528 (2000).

69. So, Y. S., Lee, D. G., Idnurm, A., Ianiri, G. \& Bahn, Y. S. The TOR pathway plays pleiotropic roles in growth and stress responses of the fungal pathogen Cryptococcus neoformans. Genetics 212, 1241-1258 (2019).

70. Stolz, L. E., Huynh, C. V., Thorner, J. \& York, J. D. Identification and characterization of an essential family of inositol polyphosphate 5phosphatases (INP51, INP52 and INP53 gene products) in the yeast Saccharomyces cerevisiae. Genetics 148, 1715-1729 (1998).

71. Guo, S., Stolz, L. E., Lemrow, S. M. \& York, J. D. SAC1-like domains of yeast SAC1, INP52, and INP53 and of human synaptojanin encode polyphosphoinositide phosphatases. J. Biol. Chem. 274, 12990-12995 (1999)

72. Jiang, J. et al. A type $2 \mathrm{C}$ protein phosphatase FgPtc3 is involved in cell wall integrity, lipid metabolism, and virulence in Fusarium graminearum. PLoS ONE 6, e25311 (2011).

73. Ko, Y. J. et al. Remodeling of global transcription patterns of Cryptococcus neoformans genes mediated by the stress-activated HOG signaling pathways. Eukaryot. Cell 8, 1197-1217 (2009).

74. Park, H. S. et al. Calcineurin targets involved in stress survival and fungal virulence. PLoS Pathog. 12, e1005873 (2016).

75. Taylor, G. S., Maehama, T. \& Dixon, J. E. Myotubularin, a protein tyrosine phosphatase mutated in myotubular myopathy, dephosphorylates the lipid second messenger, phosphatidylinositol 3-phosphate. Proc. Natl Acad. Sci. USA 97, 8910-8915 (2000). 
76. Parrish, W. R., Stefan, C. J. \& Emr, S. D. Essential role for the myotubularinrelated phosphatase Ymrlp and the synaptojanin-like phosphatases Sjl2p and $\mathrm{Sjl3p}$ in regulation of phosphatidylinositol 3-phosphate in yeast. Mol. Biol. Cell 15, 3567-3579 (2004).

77. Hartwell, L. H., Culotti, J. \& Reid, B. Genetic control of the cell-division cycle in yeast. I. Detection of mutants. Proc. Natl Acad. Sci. USA 66, 352-359 (1970).

78. Vazquez, H. M., Vionnet, C., Roubaty, C. \& Conzelmann, A. Cdc1 removes the ethanolamine phosphate of the first mannose of GPI anchors and thereby facilitates the integration of GPI proteins into the yeast cell wall. Mol. Biol. Cell 25, 3375-3388 (2014).

79. Keogh, M. C. et al. A phosphatase complex that dephosphorylates gammaH2AX regulates DNA damage checkpoint recovery. Nature 439, 497-501 (2006).

80. O’Neill, B. M. et al. Pph3-Psy2 is a phosphatase complex required for Rad53 dephosphorylation and replication fork restart during recovery from DNA damage. Proc. Natl Acad. Sci. USA 104, 9290-9295 (2007).

81. Jung, K. W. et al. Unraveling fungal radiation resistance regulatory networks through the genome-wide transcriptome and genetic analyses of Cryptococcus neoformans. mBio 7, e01483-16 (2016).

82. Jung, K. W. et al. Rad53- and Chk1-dependent DNA damage response pathways cooperatively promote fungal pathogenesis and modulate antifungal drug susceptibility. mBio 10, e01726-18 (2019).

83. Srivastav, R., Sharma, R., Tandon, S. \& Tandon, C. Role of DHH superfamily proteins in nucleic acids metabolism and stress tolerance in prokaryotes and eukaryotes. Int. J. Biol. Macromol. 127, 66-75 (2019).

84. Beck, T. \& Hall, M. N. The TOR signalling pathway controls nuclear localization of nutrient-regulated transcription factors. Nature 402, 689-692 (1999).

85. Jacinto, E., Guo, B., Arndt, K. T., Schmelzle, T. \& Hall, M. N. TIP41 interacts with TAP42 and negatively regulates the TOR signaling pathway. Mol. Cell $\mathbf{8}$, 1017-1026 (2001).

86. Jouvet, N., Poschmann, J., Douville, J., Marrakchi, R. \& Ramotar, D. RNA polymerase II degradation in response to rapamycin is not mediated through ubiquitylation. Biochem. Biophys. Res. Commun. 413, 248-253 (2011).

87. Steidle, E. A. et al. A novel inositol pyrophosphate phosphatase in Saccharomyces cerevisiae: Siw14 protein selectively cleaves the beta-phosphate from 5-diphosphoinositol pentakisphosphate (5PP-IP 5 ). J. Biol. Chem. 291, 6772-6783 (2016).

88. $\mathrm{Li}, \mathrm{C}$. et al. Identification of a major $\mathrm{IP}_{5}$ kinase in Cryptococcus neoformans confirms that PP-IP $\mathrm{I}_{5} / \mathrm{IP}_{7}$, not $\mathrm{IP}_{6}$, is essential for virulence. Sci. Rep. 6, 23927 (2016).

89. Perfect, J. R., Tenor, J. L., Miao, Y. \& Brennan, R. G. Trehalose pathway as an antifungal target. Virulence 8, 143-149 (2017).

90. Miao, Y. et al. Structures of trehalose-6-phosphate phosphatase from pathogenic fungi reveal the mechanisms of substrate recognition and catalysis. Proc. Natl Acad. Sci. USA 113, 7148-7153 (2016).

91. Wang, H., Gu, C., Rolfes, R. J., Jessen, H. J. \& Shears, S. B. Structural and biochemical characterization of Siw14: a protein-tyrosine phosphatase fold that metabolizes inositol pyrophosphates. J. Biol. Chem. 293, 6905-6914 (2018).

92. Chung, V. et al. Safety, tolerability, and preliminary activity of LB-100, an inhibitor of protein phosphatase $2 \mathrm{~A}$, in patients with relapsed solid tumors: An open-label, dose escalation, first-in-human, phase I trial. Clin. Cancer Res. 23, 3277-3284 (2017).

93. Lee, Y. et al. In vitro and in vivo assessment of FK506 analogs as novel antifungal drug candidates. Antimicrob. Agents Chemother. 62, e01627-18 (2018).

94. Beom, J. Y. et al. Biosynthesis of nonimmunosuppressive FK506 analogues with antifungal activity. J. Nat. Prod. 82, 2078-2086 (2019).

95. Juvvadi, P. R. et al. Harnessing calcineurin-FK506-FKBP12 crystal structures from invasive fungal pathogens to develop antifungal agents. Nat. Commun. 10, 4275 (2019).

96. Rios, P., Nunes-Xavier, C. E., Tabernero, L., Kohn, M. \& Pulido, R. Dualspecificity phosphatases as molecular targets for inhibition in human disease. Antioxid. Redox Signal 20, 2251-2273 (2014).

97. Mukhopadhyay, A. et al. Direct interaction between the inhibitor 2 and ceramide via sphingolipid-protein binding is involved in the regulation of protein phosphatase 2A activity and signaling. FASEB J. 23, 751-763 (2009).

98. Dobrowsky, R. T., Kamibayashi, C., Mumby, M. C. \& Hannun, Y. A. Ceramide activates heterotrimeric protein phosphatase 2A. J. Biol. Chem. 268, 15523-15530 (1993).

99. Jung, K. W., Lee, K. T., So, Y. S. \& Bahn, Y. S. Genetic manipulation of Cryptococcus neoformans. Curr. Protoc. Microbiol. 50, e59 (2018).

100. O’Meara, T. R. et al. The Cryptococcus neoformans Rim101 transcription factor directly regulates genes required for adaptation to the host. Mol. Cell. Biol. 34, 673-684 (2014).

101. Littman, M. L. Capsule synthesis by Cryptococcus neoformans. Trans. N. Y. Acad. Sci. 20, 623-648 (1958).

102. Thak, E. J., Kim, J., Lee, D. J., Kim, J. Y. \& Kang, H. A. Structural analysis of $\mathrm{N}$-/O-glycans assembled on proteins in yeasts. J. Microbiol. 56, 11-23 (2018).
103. Jung, K. W., Kim, S. Y., Okagaki, L. H., Nielsen, K. \& Bahn, Y. S. Ste50 adaptor protein governs sexual differentiation of Cryptococcus neoformans via the pheromone-response MAPK signaling pathway. Fungal Genet. Biol. 48, 154-165 (2011).

104. Cheon, S. A. et al. Unique evolution of the UPR pathway with a novel bZIP transcription factor, Hxll, for controlling pathogenicity of Cryptococcus neoformans. PLoS Pathog. 7, e1002177 (2011).

105. Livak, K. J. \& Schmittgen, T. D. Analysis of relative gene expression data using real-time quantitative PCR and the $2^{-\Delta \Delta \mathrm{Ct}}$ method. Methods 25, 402-408 (2001).

106. Vu, K., Weksler, B., Romero, I., Couraud, P. O. \& Gelli, A. Immortalized human brain endothelial cell line hCMEC/D3 as a model of the blood-brain barrier facilitates in vitro studies of central nervous system infection by Cryptococcus neoformans. Eukaryot. Cell 8, 1803-1807 (2009).

107. Santiago-Tirado, F. H., Onken, M. D., Cooper, J. A., Klein, R. S. \& Doering, T. L. Trojan horse transit contributes to blood-brain barrier crossing of a eukaryotic pathogen. mBio 8, e02183-02116 (2017).

108. Choi, J. et al. CFGP 2.0: a versatile web-based platform for supporting comparative and evolutionary genomics of fungi and Oomycetes. Nucleic Acids Res. 41, D714-D719 (2013).

109. Inglis, D. O. et al. Literature-based gene curation and proposed genetic nomenclature for Cryptococcus. Eukaryot. Cell 13, 878-883 (2014).

\section{Acknowledgements}

This work was supported by the Strategic Initiative for Microbiomes in Agriculture and Food funded by the Ministry of Agriculture, Food and Rural Affairs (grant 916006-2 and 918012-4 to Y.-S.B.) and, in part, by National Research Foundation of Korea grants (grants 2016R1E1A1A01943365 and 2018R1A5A1025077 to Y.-S.B.; 2018R1C1B6009031 to K.-T.L.) from the Ministry of Science and ICT, and Brain Korea 21 (BK21) PLUS programme. This work was also supported by NIH/NIAID R37 MERIT award AI3911521 and NIH/NIAID RO1 award AI50113-15 to J.H. J.H. is co-director and fellow CIFAR programme Fungal Kingdom: Threats \& Opportunities.

\section{Author contributions}

Y.-S.B. conceived the project. J.-H.J., K.-T.L., J.H., D.L., E.-H.J., J.-Y.K., Y.L., S.-H.L., Y.-S.S., K.-W.J., D.-G.L., E.J., M.L., Y.-B.J., Y.C., M.H.L., J.-S.K., S.-R.Y., J.-T.C., J.-W.L., H.C., S.-W.K., K.J.S., Y.L., E.J.T., J.C., and A.F.A. performed experiments and analysed the data. Y.-H.L., J.H., H.A.K., E.C., and Y.-S.B. supervised the experimental analysis. J.-H.J., K.-T.L., Y.-H.L., J.H., H.A.K., E.C., and Y.-S.B. wrote the manuscript. All authors have reviewed and approved this manuscript.

\section{Competing interests}

The authors declare no competing interests.

\section{Additional information}

Supplementary information is available for this paper at https://doi.org/10.1038/s41467020-18028-0.

Correspondence and requests for materials should be addressed to Y.-S.B.

Peer review information Nature Communications thanks Gustavo Goldman, Humberto Martín and the other, anonymous, reviewers for their contribution to the peer review of this work. Peer reviewer reports are available.

Reprints and permission information is available at http://www.nature.com/reprints

Publisher's note Springer Nature remains neutral with regard to jurisdictional claims in published maps and institutional affiliations.

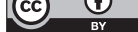

Open Access This article is licensed under a Creative Commons Attribution 4.0 International License, which permits use, sharing, adaptation, distribution and reproduction in any medium or format, as long as you give appropriate credit to the original author(s) and the source, provide a link to the Creative Commons license, and indicate if changes were made. The images or other third party material in this article are included in the article's Creative Commons license, unless indicated otherwise in a credit line to the material. If material is not included in the article's Creative Commons license and your intended use is not permitted by statutory regulation or exceeds the permitted use, you will need to obtain permission directly from the copyright holder. To view a copy of this license, visit http://creativecommons.org/ licenses/by/4.0/.

(C) The Author(s) 2020 